Cite this: Soft Matter, 2014, 10, 4885

\title{
Stretching and folding of 2-nanometer hydrocarbon rods $\uparrow+$
}

\author{
Nils O. B. Lüttschwager and Martin A. Suhm* \\ Linear alkanes $\mathrm{C}_{n} \mathrm{H}_{2 n+2}$ in vacuum isolation are finite models for an infinite polyethylene chain. Using \\ spontaneous Raman scattering in supersonic jet expansions for $n=13-21$ in different spectral ranges, \\ we determine the minimal chain length $n_{\mathrm{h}}$ for the cohesion-driven folding of the preferred extended all- \\ trans conformation into a hairpin structure. We treat fully stretched all-trans alkanes as molecular \\ "nanorods" and derive Young's modulus $E$ for the stretching of an isolated single-strand polyethylene \\ fibre by extrapolating the longitudinal acoustic mode to infinite chain length. Two key quality parameters \\ for accurate intra- and intermolecular force fields of hydrocarbons ( $\left.n_{\mathrm{h}}=18 \pm 1, E=305 \pm 5 \mathrm{GPa}\right)$ are \\ thus derived with high accuracy from experimental spectroscopy.
}

Received 6th March 2014

DOI: $10.1039 / \mathrm{c} 4 \mathrm{sm} 00508 \mathrm{~b}$

www.rsc.org/softmatter

\section{Introduction}

Any molecular description of chain polymers and their mechanical properties must address at least three energy aspects - the response to axial stress which controls the limiting elasticity, ${ }^{1}$ the torsional energy landscape of the backbone which controls the intrinsic coiling preference, ${ }^{2}$ and the interchain attraction due to non-covalent forces ${ }^{3}$ which controls general cohesion and softness. As a consequence, the quality of a given molecular mechanics force field depends on its covalent bond force constants, ${ }^{4}$ on its sterically dominated ${ }^{5}$ torsional potentials and on its van der Waals attraction terms. ${ }^{6}$ There is a wealth of such force fields in the literature ${ }^{7}$ which have been adjusted to an even larger wealth of quantum-chemical ${ }^{2,8}$ and experimental constraints. ${ }^{\mathbf{9 1 0}}$ While the former most successfully address isolated molecules, the latter often include condensed phase input. It would be desirable to have a common meeting point in the gas phase which brings theory and experiment together with respect to all three key ingredients for polymer mechanics listed above. This is the goal of the present work, focussing on the simplest of all organic chain polymers polyethylene or $-\left(\mathrm{CH}_{2}\right)_{\infty}-$.

Its stable finite fragments, saturated linear hydrocarbons or alkanes $\mathrm{CH}_{3}-\left(\mathrm{CH}_{2}\right)_{n-2}-\mathrm{CH}_{3}$, are flexible chain molecules. They have an energetical preference for the all-trans planar zig-zag conformation $\left(180^{\circ}\right.$ torsion angle). Torsion around some of the $n-3$ inner $\mathrm{C}-\mathrm{C}$ bonds by approximately $\pm 120^{\circ}$ generates an exponentially growing number of molecular conformations,

Institut für Physikalische Chemie, Universität Göttingen, Tammannstr. 6, 37077 Göttingen, Germany.E-mail: msuhm@gwdg.de

$\dagger$ This work was supported by the DFG (Su 121/2).

\$ Electronic supplementary information (ESI) available. See DOI: 10.1039/c4sm00508b with an average penalty of $\approx k_{\mathrm{B}} T_{\mathrm{R}}$ for each gauche angle at room temperature $T_{\mathrm{R}} \approx 300 \mathrm{~K}$. Some torsions are more repulsive, others even attractive due to their promotion of London dispersion interactions between the chain ends., ${ }^{2,11}$ Already for $n=5$, the unique all-trans structure ceases to prevail at room temperature. $^{8}$ This entropic disadvantage of the global minimum structure accentuates for longer chains, both in the gas phase and in the liquid state. In the solid, packing forces still favor the all-trans structure, even up to very long chain lengths. ${ }^{12}$ The resulting entropy penalty leads to record values for the enthalpy of fusion, which are exploited in phase change materials for heat storage, ${ }^{\mathbf{1 3}}$ e.g. for the stabilization of office temperatures by incorporation of such material into the walls.

The conformational dynamics of such hydrocarbon chains is decisive for the physical properties of polyethylene, the most abundant synthetic polymer. ${ }^{14}$ Its elastic modulus varies over many orders of magnitude, depending on whether the stress is acting along a chain axis (e.g. for single molecule filaments) or along entangled random coils. It is largely controlled by the degree of crystallinity. ${ }^{15}$ A single stretched polyethylene filament defines the maximum achievable elastic modulus of polyethylene material for small deformations. This limit has been subject to considerable debate. ${ }^{\mathbf{1 6 - 2 0}}$ Elaborate corrections are necessary when it is derived from condensed phase data of alkanes, ${ }^{19}$ and an isolated molecule benchmark was overdue. ${ }^{21}$

By lowering the temperature, one can gradually move from random coil character to the stretched all-trans form even for an isolated hydrocarbon molecule, if one manages to circumvent aggregation and still overcome the sizeable $\left(\approx 3 k_{\mathrm{B}} T_{\mathrm{R}}\right)$ barriers $^{\mathbf{1 1}}$ between the conformations. These requirements appear to be mutually exclusive, because the former involves rapid quenching and the latter slow cooling. There have been few strategies to straighten flexible hydrocarbon chains into stretched-out alltrans nanorods in vacuum isolation, towards the goal of 
modelling an isolated infinite polyethylene string. ${ }^{21}$ Indeed, there are several theoretical predictions that this stretched conformation will not be stable towards London dispersiondriven folding beyond a quite moderate chain length. ${ }^{7,22-24} \mathrm{Up}$ to very recently, ${ }^{25}$ not a single attempt was directed towards experimentally characterizing the critical chain length $n_{\mathrm{h}}$ at which a stretched hydrocarbon nanorod will become energetically less stable than a "hairpin" conformation, in which the two ends of the carbon chain align with each other to maximize their attraction at the expense of conformational bending energy in the hairpin fold (see Fig. 1). In view of the challenges in accurately modelling London dispersion forces, ${ }^{26}$ this represents an important gap and nonpolar hydrocarbons are well suited objects to close this gap.

The purpose of the present contribution is to elaborate on the two preliminary studies of the elastic modulus of an infinite stretched polyethylene chain ${ }^{21}$ and the critical chain length for London dispersion-driven folding, ${ }^{25}$ moving substantially beyond the experimental evidence communicated before and underpinning it with systematic and rigorous spectroscopic modelling.

\section{Experimental techniques}

Raman spectroscopy is frequently used to characterize the conformation of hydrocarbons in the condensed phase ${ }^{27-29}$ and less frequently in the gas phase, ${ }^{\mathbf{1 0}, 30,31}$ where one quickly runs out of vapor pressure with increasing $n \cdot{ }^{21}$ Low translational and rotational temperatures can be achieved by collisions in supersonic jets emerging from nozzles. ${ }^{32,33}$ These nozzles can be cylindrically symmetric or slit-like. Slit nozzles ${ }^{\mathbf{3 4 , 3 5}}$ restrict the expansion into vacuum to two dimensions, thus reducing the density fall-off and increasing the number of collisions. For low vapor pressure compounds like long chain alkanes, the gas and the expansion nozzle have to be heated, ${ }^{36,37}$ partially counteracting the cooling extent. Dilution of the compound in a light carrier gas helps to further reduce the rotational temperature.

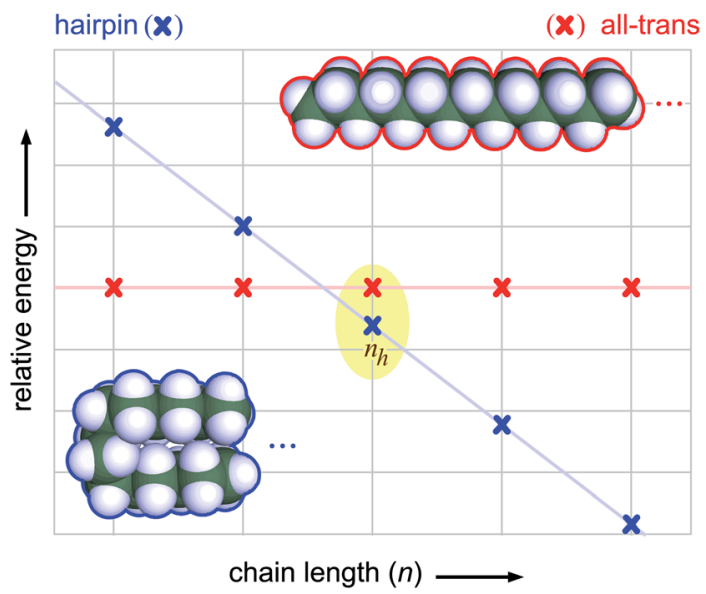

Fig. 1 Cartoon of the energy of a folded hairpin conformer relative to the all-trans conformer as a function of chain length $n . n_{\mathrm{h}}$ marks the first preference for a hairpin structure.
Vibrational cooling is less efficient, requiring more inert gas collisions than rotational relaxation. This is also true for conformational cooling, ${ }^{38}$ because the associated isomerization barriers prevent equilibration below a threshold internal energy, thus leading to a partially frozen conformational ensemble. One remedy consists in adding a small fraction of polarizable inert atoms or molecules to the expansion. Besides the kinematic mass effects of such heavier collision partners, they can transiently dock on the molecules of interest and bring in sufficient aggregation energy to overcome the conformational barriers. ${ }^{39,40}$ After a downhill conformational transition, the released excess kinetic energy can expel the inert atom or molecule, leaving behind the molecule of interest in a lower conformational state. If the temperatures are too low (nozzle distance or stagnation pressure too large), the relaxation additives may also condense on the alkane molecules or the alkanes may self-aggregate. For these reasons, the expansions used in the present work only involve $0.01-0.02 \%$ alkane in $0.5-1$ bar noncondensing helium. In some cases, $4 \% \mathrm{CF}_{4}$ is added to enhance the conformational relaxation. Ground state conformation enhancements of up to $60 \%$ are observed with $\mathrm{CF}_{4}$, but more typical values are $20-40 \%$. Achievable alkane conformational temperatures range between 100 and $200 \mathrm{~K}$ (vide infra), being lower for single barrier crossings and for the larger species. In all instances, concentrations and nozzle temperatures are chosen such that aggregation of alkanes is negligible, to avoid an influence on the conformational preference or on the band position.

For the spontaneous Raman scattering experiment ${ }^{\mathbf{2 1 , 4 1 , 4 2}}$ (Fig. 2), the expansion zone is crossed by a mildly focussed $18 \mathrm{~W}$ single mode $532 \mathrm{~nm}$ laser (Coherent) about $1 \mathrm{~mm}$ downstream a $4 \times 0.15 \mathrm{~mm}^{2}$ stainless steel slit nozzle opening to a mechanically pumped vacuum chamber. The scattered radiation is collimated perpendicularly to the flow direction and to the laser vector by a camera lens and focussed on the slit of a $1 \mathrm{~m}$ monochromator (McPherson) shielded with an ultrasteep OD 6 long pass edge filter (down to $60 \mathrm{~cm}^{-1}$ from the Rayleigh line). The monochromator is equipped with a liquid- $\mathrm{N}_{2}$ cooled CCD camera (Princeton Instruments, back-illuminated, $1340 \times 400$ pixels, full vertical binning of the slit image). Acquisition times between 1 and $10 \mathrm{~min}$ ensure that readout noise is not critical despite a photon flux as low as 1 per pixel per hour and the camera cooling minimizes the dark current. Cosmic ray signals are removed semi-automatically by iteratively comparing at least four independently recorded spectra and removing outlier pixels. Atomic transitions of $\mathrm{Ne}$ are used for spectral calibration. 7-9-point Savitzky-Golay filtering is applied to most of the spectra shown.

The gas mixtures are prepared by pre-mixing $\left(\mathrm{He}+\mathrm{CF}_{4}\right)$, by room-temperature carrier gas pickup from cooled liquid samples $^{21}$ (short alkanes), and by bubbling the pre-heated gas through a Viton-sealed brass saturator (long alkanes). The latter is kept at up to $400 \mathrm{~K}$ and supply tubes at slightly higher temperatures to avoid condensation on the way to the nozzle, which is heated up to $420 \mathrm{~K}$ but usually kept at $400 \pm 10 \mathrm{~K}$ unless stated otherwise. A sketch of the curry-jet and the brass saturator for long chain alkanes is shown in Fig. 2 (curry-jet is an acronym for "classical unrestricted Raman spectroscopy in a jet”, honoring the home country of Raman). 


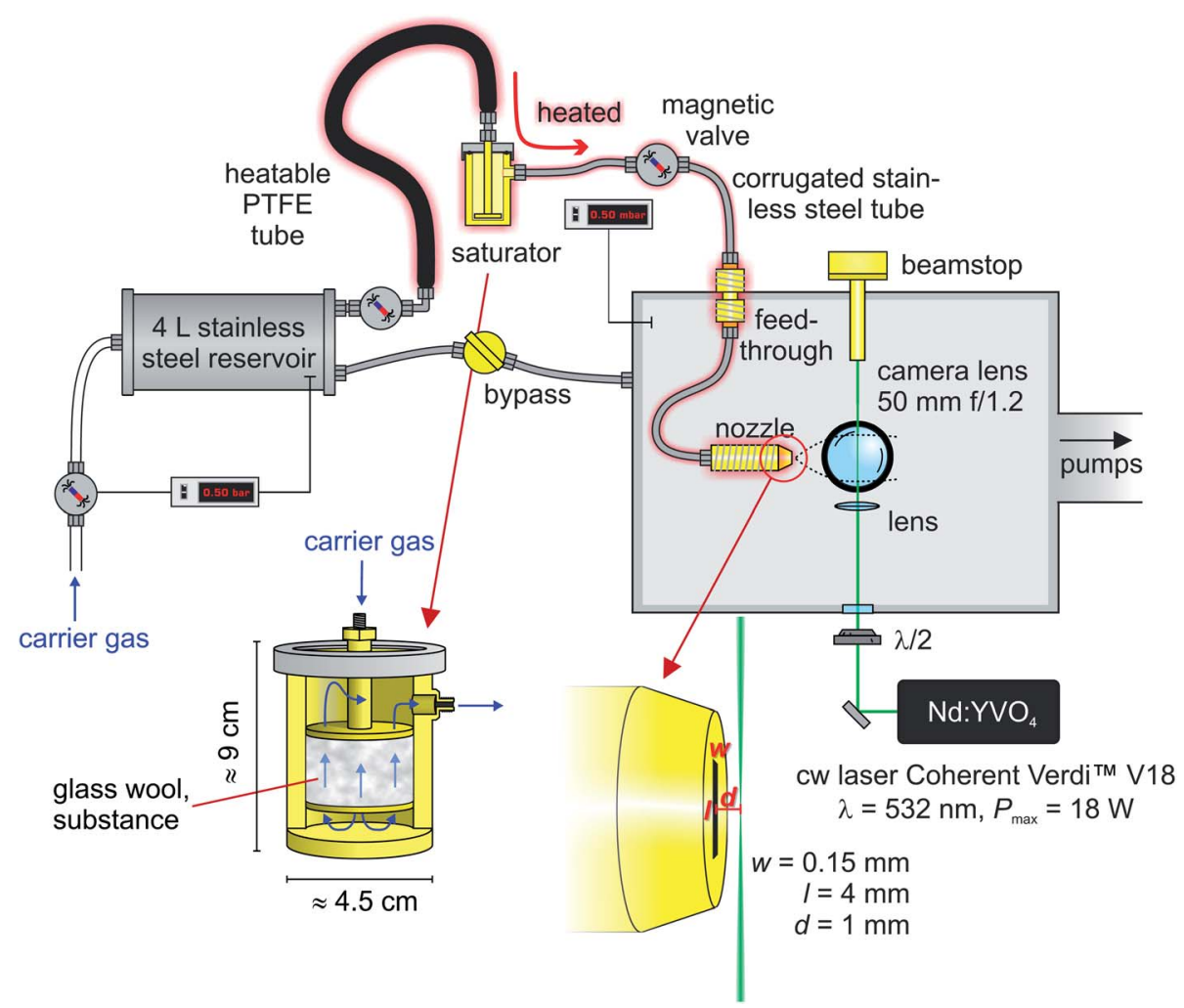

Fig. 2 Sketch of the curry-jet setup. Heating is provided by resistance heating cables or wires, wrapped around the components.

The Stokes Raman spectra have to be recorded in segments of about $500-700 \mathrm{~cm}^{-1}$. This work concentrates on the $\mathrm{CH}$ stretching range, the CC stretching and the CCC bending range. Concerted CCC bending leads to the longitudinal acoustic branch. Longitudinal acoustic modes (LAMs) offer the most selective detection of stretched or all-trans segments ${ }^{43}$ in hydrocarbons and their wavenumber is related to the chain length via the elastic modulus. ${ }^{21,44}$ For these strongly Ramanactive modes, the close vicinity to the Rayleigh line mandates particular efforts in stray light rejection.

Only relative (differential) Raman scattering cross sections $\sigma^{\prime}$ (area per solid angle) are of relevance in this work. For the setup in Fig. 2, laser polarization perpendicular to the scattering plane and isotropic molecular orientation, they can be predicted in the double-harmonic approximation (linear restoring force and linear dependence of the polarizability on vibrational normal coordinates) from calculated tensor invariants $a^{\prime}$ (change in average polarizability) and $\gamma^{\prime}$ (change in anisotropy) provided for each normal mode $k$ by the quantum chemistry software packages Gaussian 09 (ref. 45) and Turbomole v6.4. ${ }^{46}$ For photon counting of the radiation scattered by a nondegenerate vibration $\tilde{\nu}_{\mathrm{k}}$, the following expression applies: ${ }^{47}$

$$
\sigma_{\mathrm{k}}^{\prime}=\frac{2 \pi^{2} h}{c \tilde{\nu}_{\mathrm{k}}} \frac{\left(\tilde{\nu}-\tilde{\nu}_{\mathrm{k}}\right)^{3} \tilde{\nu}}{1-\exp \left(-\frac{h c \tilde{\nu}_{\mathrm{k}}}{k_{\mathrm{B}} T}\right)}\left(\frac{45 a_{\mathrm{k}}^{\prime 2}+4 \gamma_{\mathrm{k}}^{\prime 2}}{45}+\frac{\gamma_{\mathrm{k}}^{\prime 2}}{15}\right)
$$

The two last terms in parentheses correspond to perpendicular and parallel polarization of the scattering, respectively.
$T$ is the (estimated) effective vibrational temperature for low wavenumber modes, where excited states are populated and assumed to scatter at the same Stokes wavenumber as the ground state. It is relevant for the LAM intensities, but variations by a factor of two in temperature and wavenumber lead to relative intensity errors of less than $20 \%$. The $\sigma^{\prime}$ values are further corrected for the polarization-dependent efficiency of the grating, which was determined experimentally. Depending on the vibrational wavenumber, the perpendicular component is detected $50-130 \%$ more efficiently than the parallel component. Therefore, polarized (totally symmetric) vibrations and Q-branches are magnified in experimental spectra.

\section{Conformational models}

Torsion around a single C-C bond by an angle $\tau$ drives through minima separated by about $120^{\circ}$ and barriers in between due to eclipsed arrangements of the substituents. Except for the outermost $\mathrm{CH}_{3}-\mathrm{C}$ bonds, this leads to conformational isomerism. The low energy conformations are denoted $t$ for trans $\left(\tau \approx \pm 180^{\circ}\right)$ and $g^{ \pm}$for \pm gauche $\left(\tau \approx \pm 65^{\circ}\right)$. Neighbouring gauche conformations of opposite sign (syn-pentane sequences) are particularly unfavorable ${ }^{2}$ and will be neglected in our simple model. ${ }^{48}$ This is justified by their relative energy of about $12 \mathrm{~kJ}$ mol $^{-1}$ (ref. 2 and 8) and supported by Raman jet spectra of $n$ pentane (not shown). Nevertheless, the number of formally conceivable $n$-alkane conformations grows quickly with chain length, ${ }^{49}$ approaching $10^{7}$ for the longest alkanes investigated in 
this work. Several of these are still unfavorable, but instead of explicitly working out their energy within more or less sophisticated enumeration, force field or quantum chemical models, ${ }^{2,7,22,50-52}$ we take a simplified rotational isomeric statelike approach (RIS) ${ }^{53}$ adapted to the situation in the supersonic jet expansion. Consistent with the neglect of syn-pentane conformations, ${ }^{54}$ we generally neglect conformations with more than 5 gauche conformations. For isolated gauche conformations, it is reasonable to assume a uniform energy penalty relative to trans. We choose $2.5 \pm 0.5 \mathrm{~kJ} \mathrm{~mol}^{-1}$, which is close to the actual $^{10}$ energy difference for $n=4$ and 5 . Neighboring gauche conformations with the same sign definitely have lower average energy penalties due to attractive interaction between the two ends and it has therefore been suggested ${ }^{2}$ to use an average value of $2.1 \mathrm{~kJ} \mathrm{~mol}^{-1}$. The expected sparsity of gauche neighbors in a jet expansion justifies a somewhat higher value, given an appropriate error analysis. To construct a model partition function from this simplified conformational energy ladder, we further assume that the vibrational partition function is similar for all conformations and that the only significant difference in the rotational partition function relates to the symmetry number ( 2 in the presence of a two-fold rotation axis and 1 in case of less symmetric alkane conformers). One should note that chiral conformations have an additional degeneracy of 2 , such that a non-central single-gauche conformation has a four-fold statistical weight compared to the all-trans structure. This simple model holds up to $T \approx 150 \mathrm{~K}$ and $n=21$. Conformational temperatures derived from it have at least the same relative error bar as the implied uniform energy penalty, i.e. $20 \%$. Fig. 3 shows how a conformational temperature of 100 $\mathrm{K}$ translates into $n$-tuple gauche conformer fractions as a function of chain length based on our simplified approach. In principle, a more sophisticated RIS model could be applied to refine this prediction (e.g. by including higher energy rotational states $)^{55}$ but we do not expect a significant deviation for the relatively low temperature and we lack experimental data on double and higher multi-gauche conformer fractions to evaluate such a model thoroughly.

Of course, our basic model does not assign the correct approximate energy in case of a hairpin conformation, where the alkane is kinked in such a way that the two chain ends can align with each other (Fig. 1). Although there are several ways in which it can do so to optimize the inter-chain London dispersion interaction, one folding motif stands out due to its nearly strain-free realization of two neighboring parallel zig-zag strands. It consists of a sequence of four homoconfigurational (equal-signed) gauche torsions separated in the middle by a single trans conformation ( $g g t g g$ ). It profits twice from a synergetic arrangement of neighboring homoconfigurational gauche angles, itself driven by favorable dispersion interactions. ${ }^{56}$ It is also the preferred folding motif in cycloalkanes, where the chain "ends" are forced together by a covalent bond. ${ }^{57,58}$ In contrast to the stretched all-trans conformation, the ggtgg hairpin structures do not coincide with their mirror image (a case of dispersion-driven three-fold chirality synchronization ${ }^{59}$ ), also giving them a twofold or even fourfold statistical advantage in the isomer ensemble.

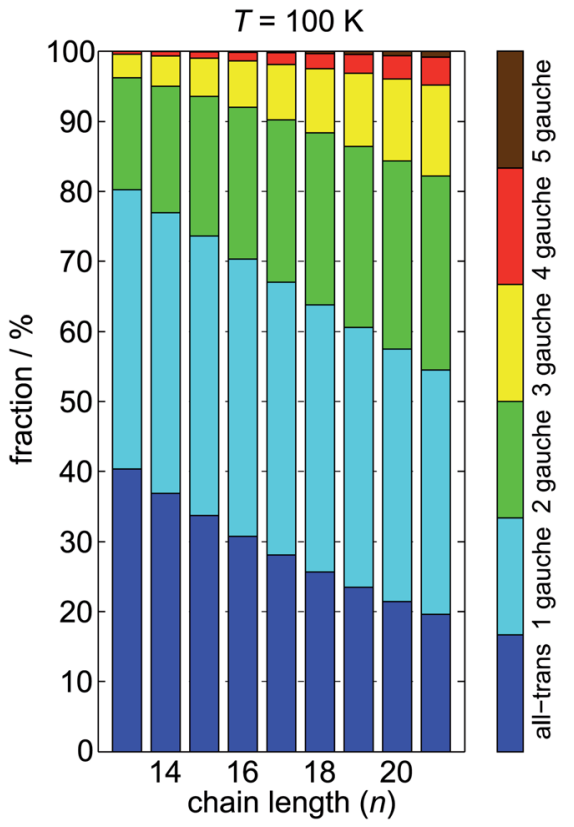

Fig. 3 Conformer fractions calculated for different chain lengths at a temperature $T=100 \mathrm{~K}$ using a model partition function with a uniform gauche energy penalty of $2.5 \mathrm{~kJ} \mathrm{~mol}^{-1}$.

\section{Spectral simulation}

To simulate alkane spectra, we take advantage of the jet cooling, which narrows down the conformational distribution to alltrans, single-gauche, and a smaller fraction of double-gauche and higher multi-gauche conformations (see Fig. 3), if a conformational temperature of $\approx 100 \mathrm{~K}$ can be achieved. This allows us to go beyond molecular mechanics, ${ }^{4}$ because only a double-digit number of important conformations have to be calculated for the set of chain lengths considered in this work ( $n$ 13-21). An alkane conformer with $n$ carbon atoms has $9 n$ normal modes, which can all be probed by either infrared or Raman spectroscopy or by both. Due to the large mass difference in the $\mathrm{CH}_{2}$ building blocks, the normal modes can be grouped into $2 n+2 \mathrm{CH}$ stretching vibrations around $3000 \mathrm{~cm}^{-1}$ and $7 n-2$ more or less coupled $\mathrm{CH}$ bending, CC-stretching, CCC-bending and all kinds of torsion motions from 0 to $1600 \mathrm{~cm}^{-1}$. The chain nature of $n$-alkanes invites a coupled oscillator treatment which yields a standing wave description of the vibrational normal modes. ${ }^{60}$ The normal modes can then be characterized by the phase shift $\varphi$ between two adjacent oscillators or the related number of nodes $m$, which ranges from 0 , a complete in-phase movement, to $N-1$, a complete out-of-phase movement of $N$ coupled oscillators. Vibrations which carry high Raman or infrared intensity have either a high or low number of nodes, in contrast to the infinite chain limit ${ }^{61}$ where only complete in-phase or out-ofphase vibrations are infrared or Raman active. Even for moderately long trans-sequences separated by gauche defects in conformationally disordered alkanes, one can often identify regularity ${ }^{62}$ besides defect modes, ${ }^{63}$ but usually the intensity spreads more evenly over several bands. ${ }^{43}$ 
If the end groups of a finite chain are negligible because the chain is long, its vibrational wavenumbers should fall close to a smooth dispersion relationship for the normal mode wavenumber as a function of phase shift in the case of an infinite stretched polyethylene chain. ${ }^{64}$ The three atoms in the methylene repeat unit generate 9 frequency branches. ${ }^{61}$ Two acoustical ones, corresponding to longitudinal CCC bending and transversal CCCC torsion, ${ }^{65}$ start at zero in the limit of an infinite chain. The strongly Raman-active longitudinal acoustic mode with one node (LAM-1) will be studied in particular detail and the one with three nodes also acquires measurable Raman intensity. Both are related to the elastic modulus of an infinite polyethylene chain. The other, more localized optical branches involve a range of $\mathrm{CH}_{2}$ bending modes and $\mathrm{CC}^{62}$ (800$\left.1100 \mathrm{~cm}^{-1}\right)$ as well as $\mathrm{CH}\left(3000 \mathrm{~cm}^{-1}\right)$ stretching fundamentals. Here, we concentrate on the stretching branches, which are sufficiently Raman active and sensitive to folding. Note that there is no $1: 1$ correspondence between the normal modes and the valence descriptions given above. If concerted CCC bending and CC stretching motions are in phase relative to each other, this gives rise to the LAM-1 mode. If they have opposite phase, a completely in-phase CC stretching mode is generated.

The spectral simulation involves structure optimization, harmonic vibrational analysis including Raman scattering cross sections and weighted summation of Gaussian-convoluted stick spectra (FWHM of $4 \mathrm{~cm}^{-1}$ and somewhat larger for hairpin structures, inspired from experimental spectra). The B3LYP hybrid density functional shows a good performance in predicting hydrocarbon vibrational wavenumbers, ${ }^{21}$ partly due to error compensation between neglected anharmonic terms and slightly too soft chemical bonds. In the low frequency range, wavenumber scaling-factors close to unity $(0.98,0.99)$ are thus used to match the experimental spectra. The B3LYP folding energetics are poor, like in other popular semilocal functionals, ${ }^{66}$ but this can be cured by adding a Grimme D3 dispersion correction on top of the electronic structure calculation. ${ }^{67}$ Besides a standard Pople $6-311++\mathrm{G}^{* *}$ basis set, the simpler $\operatorname{def}-\mathrm{SV}(\mathrm{P})$ and the computationally more expensive def2-TZVP ${ }^{68}$ basis set were explored. In each case, the energy convergence criterion was set to at least $10^{-7}$ hartree, the gradient norm convergence criterion to at least $10^{-4}$ hartree bohr $^{-1}$ and the $\mathrm{m} 4$ grid $^{69}$ was used. Differences are minor and the discussion will be restricted to 6$311++\mathrm{G}^{* *}$ for spectra and def2-TZVP for the energetics. Even for $6-311++\mathrm{G}^{* *}$, the conformational energy differences for $n=5$ and 6 never deviate by more than $6 \%$ from a high quality electronic energy benchmark. ${ }^{8}$ Raman intensities are certainly more adequate with a large basis set $^{70}$ and might profit from a consistent electron correlation approach, but for the simplified modelling relevant in the spectral assignment and given the poorly characterized vibrational temperature (assumed to be 100 $\mathrm{K}$ throughout these simulations), harmonic B3LYP-D3/6$311++\mathrm{G}^{* *}$ with dynamic polarizability as implemented in Turbomole is considered sufficient. The cost-effectiveness of the B3LYP approach for relevant Raman intensities has been emphasized in recent work. ${ }^{70,71}$

Conformational weighting is non-trivial. For single-gauche to all-trans ratios, we assume a uniform conformational
Boltzmann temperature because of the single, more or less uniform barrier for interconversion. It cannot be decided to which degree this effective temperature reflects a true equilibrium situation or a uniform freezing of conformational conversion due to inefficient or fading collisions. For the hairpin structures, which are separated by at least three barriers from the dominant single-gauche conformations, the kinetic control will dominate and their abundance has to be taken from experiment.

Beyond the simple additive $2.5 \mathrm{~kJ} \mathrm{~mol}^{-1}$ trans-gauche increment model, the quantum chemical calculations allow for an individual weighting of single-gauche abundances. Rewardingly, all single-gauche isomerization energies for $n=13-21$ including zero point energy are within $2-3 \mathrm{~kJ} \mathrm{~mol}^{-1}$ window at the B3LYP-D3/6-311++G** level. Assuming thermal equilibrium, the Boltzmann factors derived from the difference in zero point level for the conformations $\Delta E_{i j}^{0}$ could be refined by the ratio of the vibrational and rotational partition functions. The gauche-trans rotational partition function ratio is robust and exceeds the ratio of the symmetry numbers $\sigma_{i}$ (order of the rotational symmetry sub-group) by $10-60 \%$ in test calculations for $n=13$, because the trans conformation features the smallest moment of inertia. The vibrational partition function ratio is very sensitive to numerical errors in the low frequency skeletal modes, which can in some cases even lead to a single imaginary frequency for $n>17$. In the more robust case of $n=13$, the ratio is actually lower than 1 by $10-30 \%$, because the all-trans conformation contains the softest modes. Therefore, we omit these uncertain but qualitatively compensating partition function ratios from the simulation and use

$$
\frac{N_{j}}{N_{i}}=\frac{g_{j}}{g_{i}} \frac{\sigma_{i}}{\sigma_{j}} \exp \left(-\frac{\Delta E_{i j}^{0}}{R T}\right)
$$

where $N_{i}$ is the population of conformation $i$ and $g_{i}$ is its chiral degeneracy.

To underpin these approximations, Fig. 4 shows a comparison of experiment (with and without addition of $\mathrm{CF}_{4}$ to the carrier gas $\mathrm{He}$ ) and modelling for $n=13$ in the low-frequency region. The bottom traces contain unweighted B3LYP-D3/6$311++\mathrm{G}^{* *}$ stem plots for scattering cross sections of the all-trans and the five spectroscopically distinguishable single-gauche isomers as well as the energetically remote hairpin conformation, to help identify the specific conformations (single-gauche conformers are referred to according to the position of the gauche conformation in the chain, counting $\mathrm{CH}_{2}-\mathrm{CH}_{2}$ bonds from the chain end to the center). They are intensity-scaled to the all-trans LAM-1 or accordion mode and shown down to a relative intensity of $1 \%$. The wavenumber scaling factor is 0.99 . The simulation trace assumes a uniform conformational temperature of $110 \mathrm{~K}$, except for the elusive hairpin conformer which is included with $10 \%$ of the all-trans weight, to show that it is not present within experimental signal-to-noise ratio (obtained for a total Raman acquisition time of $2 \mathrm{~h}$ ). The experimental intensity pattern is well reproduced by the simulation, supporting an equilibrated or uniformly frozen single gauche/all-trans distribution. Obviously, a detailed assignment 


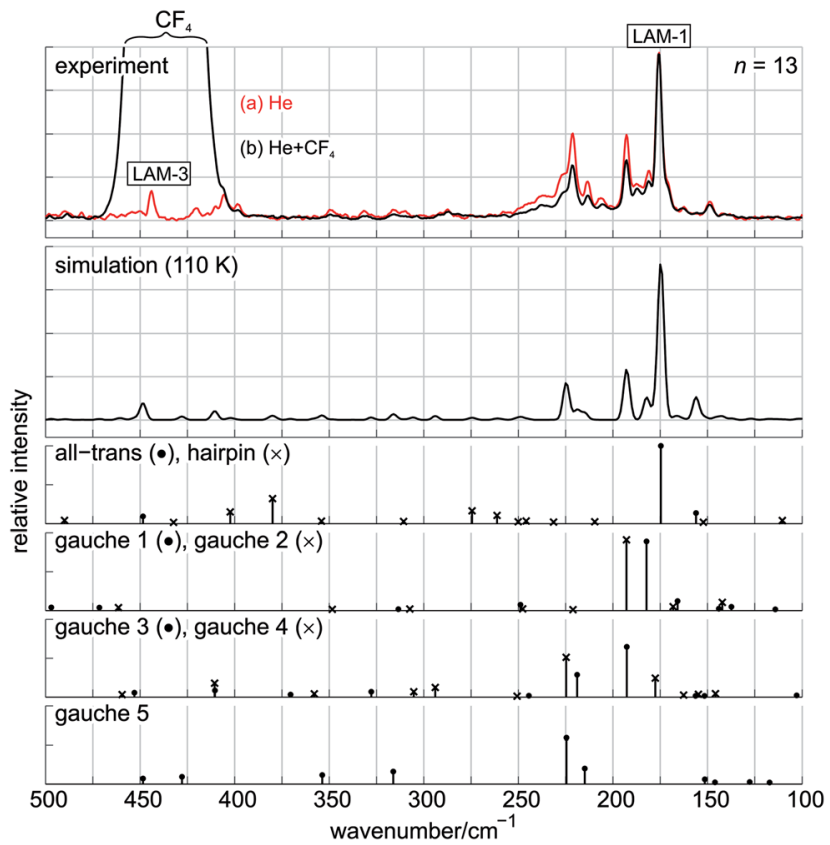

Fig. 4 Comparison of averaged jet-cooled Raman spectra of tridecane in the low-frequency region to a B3LYP-D3/6-311++G** simulation. Stem plots correspond to unweighted scattering cross sections relative to the all-trans accordion vibration (LAM-1). Only vibrations with at least $1 \%$ of the accordion vibration intensity are included.

of many observed single-gauche bands is possible, although this is not the main focus of the present work. At this point, it is appropriate to switch to the presentation and discussion of the low frequency spectra for increasing chain lengths.

\section{Low-frequency spectra}

We start with a survey of the spectra obtained for $n=16-21$ in pure He in Fig. 5. The corresponding spectra for $n=10-15$ are shown in the supplement. The sharp LAM-1 signal of the alltrans conformation is clearly seen at the right, moving to lower wavenumber with increasing chain length ${ }^{65}$ and dominating all spectra but the lowest. Occasionally, it has weak satellites due to mode mixing with energetically close transverse fundamentals (*). To the right, weak rotational signals due to air impurities may be spotted $\left(\tilde{\nu} \leq 100 \mathrm{~cm}^{-1}\right)$. They could be partially responsible for better relaxation away from the all-trans conformation in the case of $n=21$, where they are most prominent. Sharp bands at higher wavenumbers due to singlegauche conformations are much less prominent than in the case of $n=13$ and other short chain lengths, indicative of a lower effective conformational temperature. Instead, broader features start to grow around 200, 400 and $540 \mathrm{~cm}^{-1}$. The former two correspond to D-LAM signals in liquid alkanes, ${ }^{43,62}$ attributed to a superposition of multiple gauche conformations, whereas the latter appears to be specific for the jet spectra. It is reminiscent of features in the Raman spectra of cycloalkanes, ${ }^{58,63}$ where ggtgg hairpin turns are enforced and may thus be related to such structures. The onset of this band near $n=18$ already gives an

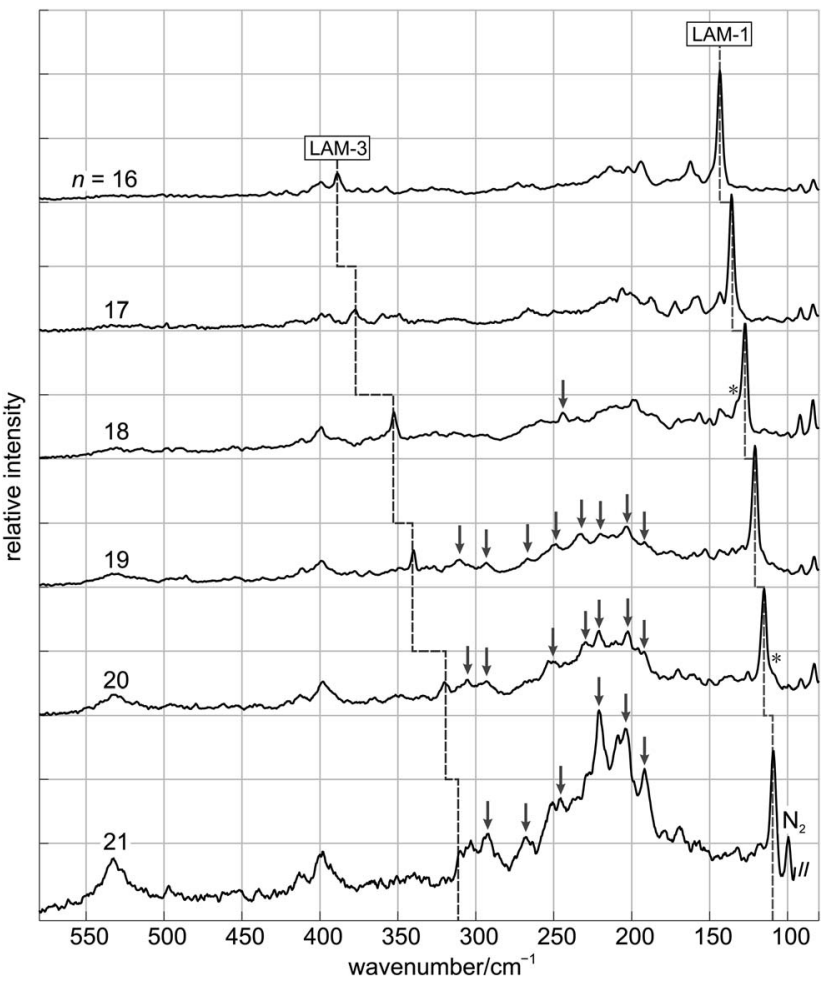

Fig. 5 Averaged jet-cooled Raman spectra of the low-frequency region of alkanes with chain lengths $n=16-21$ in He expansions. Mode mixing is considered in case of $n=18,20$ (marked with *). Bands assigned to hairpin conformers are marked with arrows. Air impurities $\left(\tilde{\nu} \leq 100 \mathrm{~cm}^{-1}\right)$ are prominent especially in the case of $n=21$.

empirical hint for the emergence of hairpin structures, possibly with some side arm disorder. Several moderately narrow bands in the $200-300 \mathrm{~cm}^{-1}$ region are marked with arrows and will be discussed more specifically in the context of regular hairpin structures.

Although each experimental spectrum between $n=13$ and $n$ $=21$ has its peculiarities which deserve discussion in the context of the spectral simulation and its quality assessment, here we concentrate on $n>15$ (Fig. 6). The shorter chains have already been addressed briefly in previous work with a roomtemperature nozzle ${ }^{21}$ and the improved experimental conditions in the present work do not provide any evidence for hairpin formation. Here we only mention one of the major discrepancies between experiment and simulation for $n=13$ (Fig. 4), the peak near $150 \mathrm{~cm}^{-1}$. It is due to a transverse acoustic mode from the same branch of the all-trans conformation as the dominant LAM-1 mode, which is evident from the same relaxation behaviour upon $\mathrm{CF}_{4}$ addition. It gains intensity from mode mixing with the accidentally close LAM-1 and this mode mixing is somewhat less pronounced in the experiment than in the simulation. Such an intensity redistribution among several modes is more frequent in symmetry-broken singlegauche conformations, as the individual stem plots illustrate.

Experimental and simulated Raman spectra for $n=16-21$ are compared in Fig. 6 ( $\mathrm{He}$ expansions in red, $\mathrm{He}+\mathrm{CF}_{4}$ expansions in black). Inverted stem plots are shown to identify all-trans and hairpin bands. Starting with $n=16$, a broad 

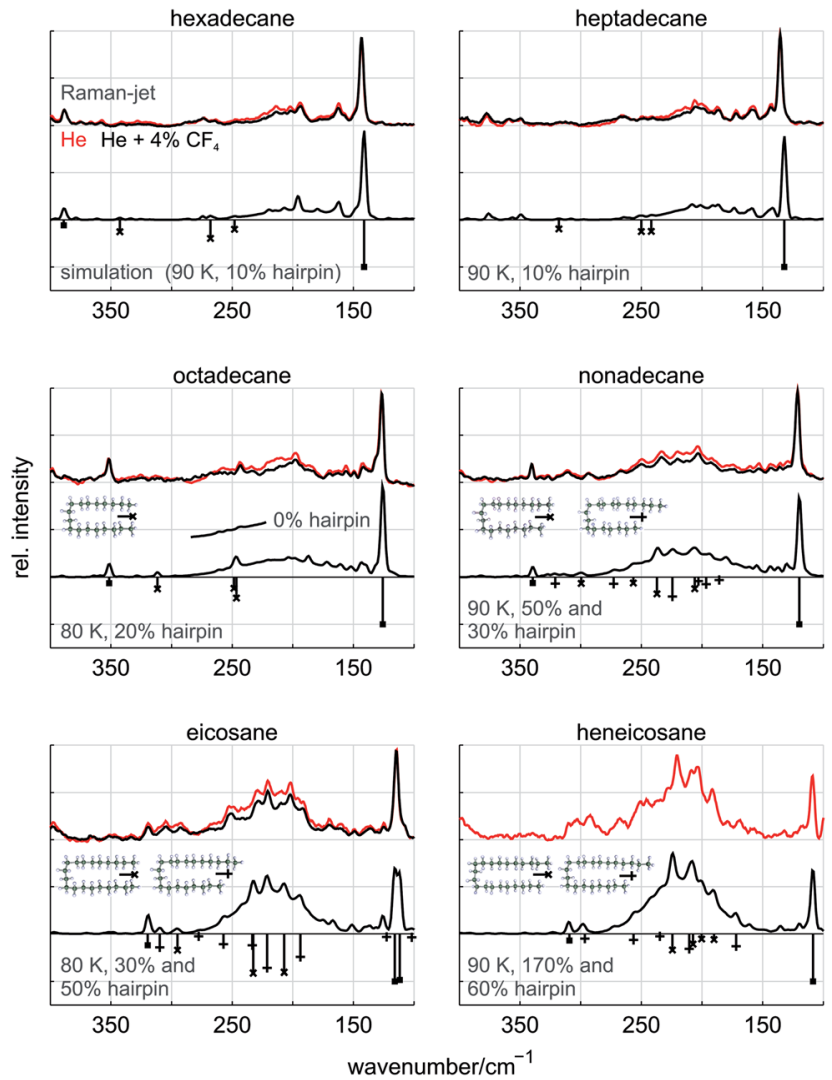

Fig. 6 Comparison of averaged jet-cooled Raman spectra of $n=16-$ 21 in the low-frequency region to B3LYP-D3/6-311++G** simulations (He expansion in red, $\mathrm{He}+\mathrm{CF}_{4}$ expansions in black). For better clarity, stem plots are restricted to sufficiently Raman active all-trans conformer (squares) and hairpin conformer vibrations (upright and tilted crosses). Simulated harmonic wavenumbers are scaled: $\tilde{\nu} \times 0.99$ $(n=16,18-21), \tilde{\nu} \times 0.98(n=17)$. Hairpin fractions in percent are relative to all-trans.

Gaussian component around $200 \mathrm{~cm}^{-1}$ with floating height and width is added to the simulation trace to visualize the combined effect of multiple gauche conformations, termed D-LAM. The alltrans conformation contributes its LAM-1 band at $143 \mathrm{~cm}^{-1}$ and a LAM-3 band at $389 \mathrm{~cm}^{-1}$. The bands at 162 and $194 \mathrm{~cm}^{-1}$ are superpositions of gauche 3/gauche 7 and gauche 5/gauche 6 conformations, respectively, according to the B3LYP calculations (the number refers to the position of the gauche conformation in the chain). The gauche 7 contribution is probably minor due to its symmetry number of 2 but this is compensated by a strong intensity and slightly higher stability predicted for gauche 3 .

The $10 \%$ hairpin signature (relative to all-trans: $N_{\text {hairpin }} / N_{\text {all-trans }}$ $=0.1$ ) simulated near $250-275 \mathrm{~cm}^{-1}$ does have an apparent experimental counterpart, but gauche $5-7$ also contribute in this range at an effective conformational temperature of $90 \mathrm{~K}$, rendering this a generous upper bound for any hairpin abundance.

The situation is quite analogous for $n=17$. Again, the alltrans conformation dominates, although the LAM-3 mode is now split due to mode-mixing with a totally symmetric TAM mode ( 378 and $350 \mathrm{~cm}^{-1}$ ). The single-gauche band pattern spreads from the LAM-1 at $136 \mathrm{~cm}^{-1}$ to about $210 \mathrm{~cm}^{-1}$, shows less overlap than $n=16$ and is modeled surprisingly well by the B3LYP-D3 approach, once the predicted wavenumbers are scaled by 0.98 . Hairpin contributions below the simulated $10 \%$ level again cannot be ruled out.

The first significant evidence for a $10-20 \%$ hairpin contribution is found for $n=18$, where the best fit of the single-gauche conformational temperature has now dropped to $80 \mathrm{~K}$. Consistent with this lower temperature, the single-gauche pattern is weaker, but very well reproduced by the quantumchemical simulation. The broad multi-gauche D-LAM band is now more prominent but shrinks with $\mathrm{CF}_{4}$ addition, as expected.

On top of it, a band located at $243 \mathrm{~cm}^{-1}$ is too strong to be explained only by single-gauche conformers and it coincides with the prediction of two overlapping hairpin bands. Therefore, simulations for $0 \%$ and $20 \%$ hairpin content are shown. However, the predicted $312 \mathrm{~cm}^{-1}$ counterpart is missing in the experiment, suggesting less than $20 \%$ hairpin content.

Turning now to $n=19$, the circumstantial hairpin evidence in the low-frequency range changes into a definitive spectroscopic signature, thanks to the high fidelity of the B3LYP-D3 spectral predictions. The D-LAM region acquires structure which correlates very closely with a variety of calculated hairpin vibrations. The simulation includes two unsymmetric hairpin structures, predicted to have their strongest Raman signals between 200 and $250 \mathrm{~cm}^{-1}$, where no strong signals from singlegauche conformers are expected. Although the simulated intensity pattern is not perfect, the best agreement is achieved by including $50 \%$ of the all-trans abundance for the more stable hairpin and $30 \%$ for the less stable hairpin $b$, each of them exceeding even the most abundant single-gauche conformer at $90 \mathrm{~K}$.

$n=20$ allows for a symmetric hairpin, which is included in the spectral simulation together with an unsymmetric variant. The apparently higher intensity of these structures in the stem plot is actually a consequence of substantial LAM-1/TAM mode mixing in the all-trans conformer, which is again (like for $n=$ 13) exaggerated by the B3LYP calculation. This renders temperature and hairpin abundance estimates less reliable in this case. In line with the symmetry number of 2 for the symmetric hairpin, it is estimated at a lower relative abundance $(30 \%)$ than the less stable but statistically favored unsymmetric one $(50 \%)$. The increasing D-LAM background adds to the difficulty of hairpin quantification, but the qualitative match between simulated and experimental peaks is again convincing.

A further boost of hairpin signals is observed for $n=21$, where air impurities in the He expansion render the conditions somewhat intermediate between $\mathrm{He}$ and $\mathrm{He} / \mathrm{CF}_{4}$. The hairpin abundance jumps to $170 \%$ and $60 \%$ of the all-trans abundance for the two considered isomers. The match of experiment and simulation is close, but missing simulated intensity at the highwavenumber slope of the D-LAM band may point at the importance of further hairpin isomers. Single-gauche contributions are very low, indicating that the conformational diversity is switching from the nearly stretched to the nearly folded regime. 
An analysis of the detected intense hairpin modes in terms of atomic displacements shows that LAM-like stretching of one or both arms is an important ingredient, thus explaining why these modes occur at approximately twice the wavenumber observed for the all-trans mode. Twisting motion and other deformations of the kink are also involved, but in some cases, the wavenumber can be reliably predicted from the length of the vibrating arm. Therefore, it may be expected that some bands reappear when the number of carbon atoms is increased in the non-vibrating arm only. Indeed, the unassigned experimental $n=21$ band at $268 \mathrm{~cm}^{-1}$ may be explained by a hairpin $\mathrm{b}$ structure for $n=19\left(267 \mathrm{~cm}^{-1}\right)$ which has been extended by two units on the non-vibrating long arm. This illustrates how the high regularity of the all-trans spectral pattern may find counterparts in the hairpin spectroscopy, once it is probed more systematically. Such an extension will also have to include isolated gauche isomerizations in the hairpin arms, to explain conformational diversity and leading the way to the paper-clip motif. ${ }^{7}$

Before turning to other spectral ranges, the regular behavior of the LAM modes as a function of hydrocarbon chain length shall be analyzed in terms of the elastic modulus of an infinite stretched polyethylene chain.

\section{Nanorod stretching}

The longitudinal elastic modulus or Young's modulus $E$ of a homogeneous rod of cross section $A$ and length $l$ is defined as the force $F$ per area $A$ (stress) required to achieve a relative elongation $\Delta l / l$ (strain) for small (harmonic) elongations:

$$
E=\frac{F / A}{\Delta l / l}
$$

In the limit of a macroscopic crystal built from densely packed bundles of infinite all-trans alkane chains, this elastic modulus can be related by its density $\rho$ and the speed of light $c_{0}$ to the LAM- $m$ mode wavenumber via: ${ }^{72}$

$$
\tilde{\nu}=\frac{m}{2 c_{0} l} \sqrt{\frac{E}{\rho}},
$$

Because such a crystal cannot be synthesized, two approximations have been previously adopted. One is to study crystalline domains in real polyethylene samples by diffraction methods $^{73}$ and the other is to study LAM wavenumbers in alkane crystals with increasing chain length and extrapolate to infinite chain length. ${ }^{\mathbf{4 , 6 5}}$ Both methods have their shortcomings and provide mutually inconsistent values. Besides polyethylene, the LAM wavenumber extrapolation was applied to various other polymers like polytetrafluoroethylene, ${ }^{74}$ polyoxymethylene, or isotactic polypropylene ${ }^{75}$ and is an accepted approach, but it suffers from neglecting longitudinal intermolecular forces if short molecules are investigated. ${ }^{19}$ Over the last decades, the gap has been narrowed down by sophisticated corrections $^{16,19}$ but it would be rewarding to have a third, independent method available. This consists in avoiding the effect of longitudinal intermolecular forces in the chain bundles on the longitudinal modes and to extrapolate the LAM modes for isolated all-trans chains to infinity. ${ }^{21}$ Although the density of a single vacuum-isolated molecule cannot be defined unambiguously, comparability to solid state investigations dictates the usage of the experimental crystal domain density $\rho$, implying close packing but negligible transverse interactions (i.e. we set the volume of a vacuum-isolated chain to the volume it would occupy in a crystal). There is consensus in the computational $^{76-78}$ and experimental ${ }^{16,19,44}$ literature to use $\rho \approx$ $1.00 \mathrm{~kg} \mathrm{dm}^{-3}$ and the corresponding space-filling cross-section $A=0.1824 \mathrm{~nm}^{2}$ from an X-ray structure determination published by Bunn. ${ }^{79}$ There is also consensus to use an experimental segment separation $d=0.1267 \mathrm{~nm}$ (ref. 79) along the chain axis to convert the atomistic chain length $n$ to the macroscopic crystal length $l$. When using finite chains capped with hydrogen atoms, the latter have to be considered. Fig. 7 shows that the segment length for the 1.7-2.7 $\mathrm{nm}$ alkanes obtained in this work at the electronic B3LYP-D3/6-311++G** level is $0.128 \mathrm{~nm}$, in good agreement with the crystallographic value. One should emphasize that the primary quantity obtainable from this work is the limit $S=\lim _{n \rightarrow \infty} n \tilde{\nu}_{n}$ at low temperature, which is independent on any macroscopic assumptions ${ }^{20,80}$ and can be converted to the elastic modulus via

$$
E=4 \rho\left(c_{0} d S\right)^{2},
$$

using preferred values for $\rho$ and $d$. Therefore, we first derive the model-independent $S$-value for the initial slope of the longitudinal acoustic branch as a function of the phase angle $\varphi / \pi$ in

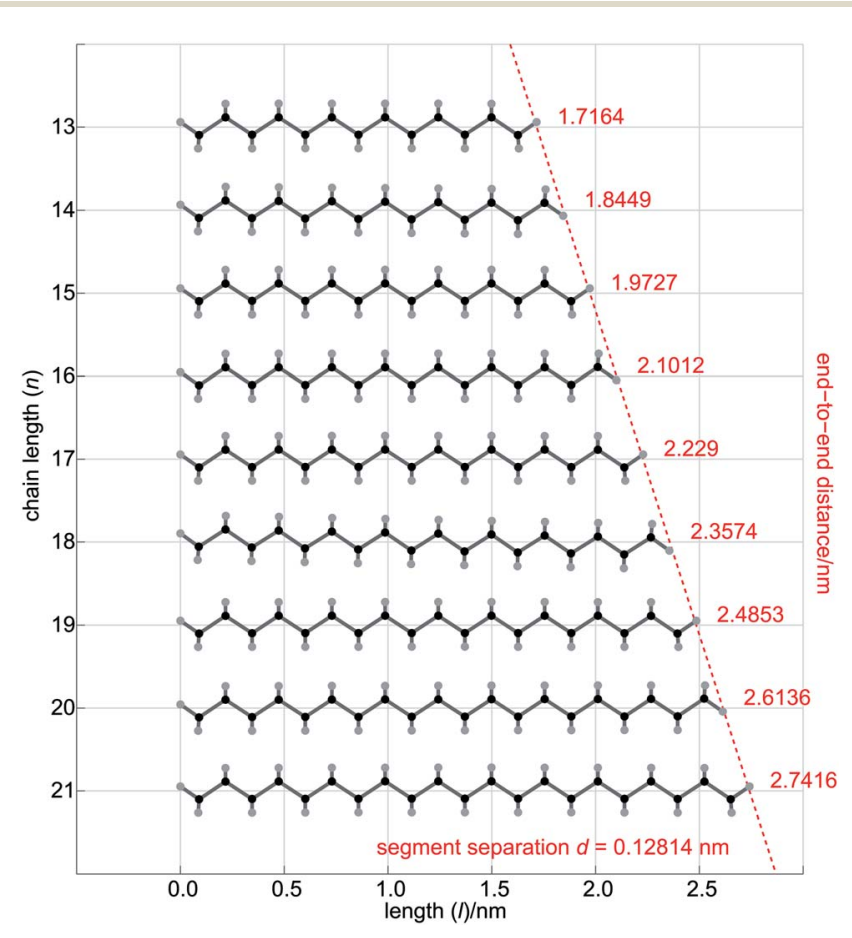

Fig. 7 Projection of all-trans alkane atom coordinates on the plane spanned by the carbon atoms (optimized structures on the B3LYP-D3/ 6-311++G** level), Turbomole v6.4 (ref. 46). 
terms of the initial slope of the LAM-1 wavenumber as a function of $m / n$.

For the extrapolation from finite $n$ to infinity, some of the LAM-1 modes have to be deperturbed from mode mixing with neighboring, intrinsically much less Raman-active low wavenumber modes of the same symmetry. ${ }^{21}$ Independent on whether the mixing is already found at the harmonic level (i.e. reproduced more or less by the B3LYP-D3 calculations) or whether it builds on anharmonic Fermi resonance with combinations of very low wavenumber modes, their deperturbation rests on evaluating the center of gravity of the Raman intensity pattern. ${ }^{21}$ For the small corrections involved, the underlying assumption of zero intrinsic intensity of the perturber is a reasonable one. Harmonic mixing is considered for $n=13,18$ and 20. For $n=18$, the perturber is obscured by single-gauche bands. The anharmonic resonance for $n=14$ was discussed before and the results of all deperturbations and unperturbed LAM bands are summarized in Table 1. The influence of band overlap with bands of other conformers on the LAM-1 position is negligible, since hairpin conformers and other multi-gauche conformers do not contribute in the LAM-1 wavenumber region with single intense bands and single-gauche bands are very small (especially for the longer alkanes). The residual wavenumber uncertainty after careful recalibration with $\mathrm{N}_{2}$ (ref. 81) and $\mathrm{CF}_{4}$ (ref. 82) transitions is estimated close to $\pm 1 \mathrm{~cm}^{-1}$.

In Fig. 8, the wavenumbers of LAMs with one and three nodes ( $m=1$ and 3 ) are plotted against $m / n$ (ref. 44 ) and LAM-1 wavenumbers are fitted in the linear range $n=16-21$ (filled circles). LAM-3 wavenumbers are included to demonstrate the flattening of the CCC bending frequency branch with decreasing $m / n$ which underpins the accuracy of a linear extrapolation of the LAM-1 data. Any remaining non-linearity which would lower the accuracy of the extrapolation is disguised by the small scattering of the data which excludes a substantial error. The overshooting $n=15$ value may be caused by a non-assigned Fermi resonance and the curvature becomes visible in the range $n=13,14$. The fitted slope is $S=2295 \pm 8$ $\mathrm{cm}^{-1}$. The systematic error due to non-linearity in the range $n=$ 16-21 may be of similar magnitude, leading to a conservative estimate of $S=2.30 \pm 0.02 \times 10^{3} \mathrm{~cm}^{-1}$. LAM-3 wavenumbers could be used for a linear extrapolation if they were available for much larger $n$.

At this stage, an infinite polyethylene chain elastic modulus $E=305 \pm 5 \mathrm{GPa}$ using the crystal density $\rho=1.00 \mathrm{~kg} \mathrm{dm}^{-3}$ and the segment length $d=0.1267 \mathrm{~nm}$ can be derived. It corresponds to a force of $(5.6 \pm 0.1) \times 10^{2} \mathrm{pN}$ required to stretch such

Table 1 Averaged wavenumbers of LAM-1 and LAM-3 bands from Raman jet measurements in $\mathrm{cm}^{-1}$. In case of tridecane, tetradecane, and eicosane, the deperturbed LAM-1 wavenumber is given in italics (see text)

\begin{tabular}{llllllllll}
$n=$ & 13 & 14 & 15 & 16 & 17 & 18 & 19 & 20 & 21 \\
\hline
\end{tabular}

\begin{tabular}{llllllllll}
\hline LAM-1 & 172.8 & 162.4 & 154.4 & 143.3 & 135.6 & 126.8 & 121.0 & 115.0 & 109.1
\end{tabular} $\begin{array}{llllllllll}\text { LAM-3 } & 444 & 433 & 398 & 389 & 377 & 352 & 341 & 321 & 310\end{array}$

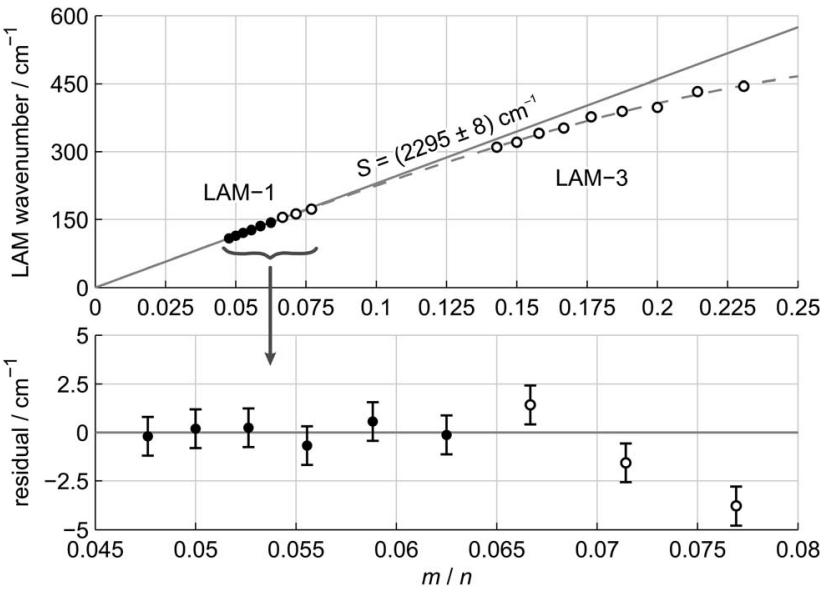

Fig. 8 Extrapolation of Raman jet LAM wavenumbers (circles) of normal alkanes $(n=13-21)$ to infinite chain length. Tridecane, tetradecane, and pentadecane LAM-1 wavenumbers as well as LAM-3 wavenumbers are excluded from the linear fit (open circles). The complete set of wavenumbers is fitted with a third order polynomial (dashed line) to guide the eye and demonstrate non-linearity in the LAM-3 region.

a molecular rod by $1 \%$ and the latter value has the advantage that it is independent on the assumed cross section of the molecular filament. This is obviously less than the force required to stretch a single $\mathrm{C}-\mathrm{C}$ bond and much more than the force required to straighten a coiled polyethylene. It represents an excellent approximation to the limiting stiffness of a perfectly ordered bundle of polyethylene strands at low temperature and thus sets an upper bound for macroscopic samples which will be affected by conformational disorder and finiteness of the chains.

The new result compares well to the previous value of $E=$ $309 \pm 8 \mathrm{GPa}$ obtained using the same experimental technique up to $n=16$ and an $a b$ initio-guided extrapolation trick, whereas the present value is purely experimental, building on experimental LAM-1 positions, experimental intensities for deperturbation, an experimental crystal density and an experimental segment length. It also compares well to a series of successively refined lamellar corrections applied to alkane crystal Raman extrapolations, starting at $364 \pm 25 \mathrm{GPa}$ (ref. 44) (corrected for the present bulk density), being improved to $290 \mathrm{GPa}$ (ref. 16) and finally to $305 \pm 3 \mathrm{GPa} .{ }^{19}$ The excellent agreement shows that lamellar corrections are under control and lateral interactions are insignificant. The highest experimentally determined real polyethylene modulus of $262 \mathrm{GPa}$ (ref. 17) at $77 \mathrm{~K}$ comes remarkably close, in particular when it is corrected to $288 \pm 10$ GPa (ref. 17) due to thermal contraction and machine softness. The X-ray diffraction approach concentrating on crystalline domains clearly yields values which are $20 \%$ too low. ${ }^{18,73}$

Quantum chemical studies often convert a calculated change of energy per strain to the elastic modulus. Elastic moduli from Hartree-Fock ${ }^{76,83}$ and pure DFT calculations ${ }^{20,78}$ not surprisingly tend to be $10-20 \%$ too large, while an early MP2 calculation almost matches the modulus reported here. ${ }^{77}$ The present value invites further quantum chemical studies of single polyethylene 
strands and crystals, eliminating the problem of thermal effects $^{80}$ and illuminating the role of interchain interactions, which certainly require dispersion corrections for an accurate description of the crystal. ${ }^{20}$

\section{CC stretching modes}

While the elastic modulus behaviour can be derived unambiguously from the LAM region, the delicate onset of hairpin formation profits from independent evidence in other spectral ranges. Alkane CC stretching modes in the spectral region from 800 to $1400 \mathrm{~cm}^{-1}$ are much less sensitive to the chain length and they are embedded in a multitude of $\mathrm{CH}$ deformation modes. Therefore, evidence for conformational changes is more delicate, but clearly detectable, as the experimental spectra (Fig. 9) and their theoretical counterparts (Fig. 10, simulated at $100 \mathrm{~K}$ single-gauche conformational temperature and including hairpin contributions deduced from the LAM region) show. Three signals which emerge around $n=18$ are marked with arrows in Fig. 9 and five simulated signals which are expected for hairpin modes are marked in Fig. 10.

The spectral assignment in this wavenumber range is well established in the literature for aggregated alkanes, ${ }^{29,84,85}$ including the $g g t g g$ sequences sought in this work. ${ }^{63}$ The cluster of bands centered at $\approx 1100 \mathrm{~cm}^{-1}$ is of particular interest because it involves two hairpin candidates. The dominating size-independent signals stem from the most in-phase

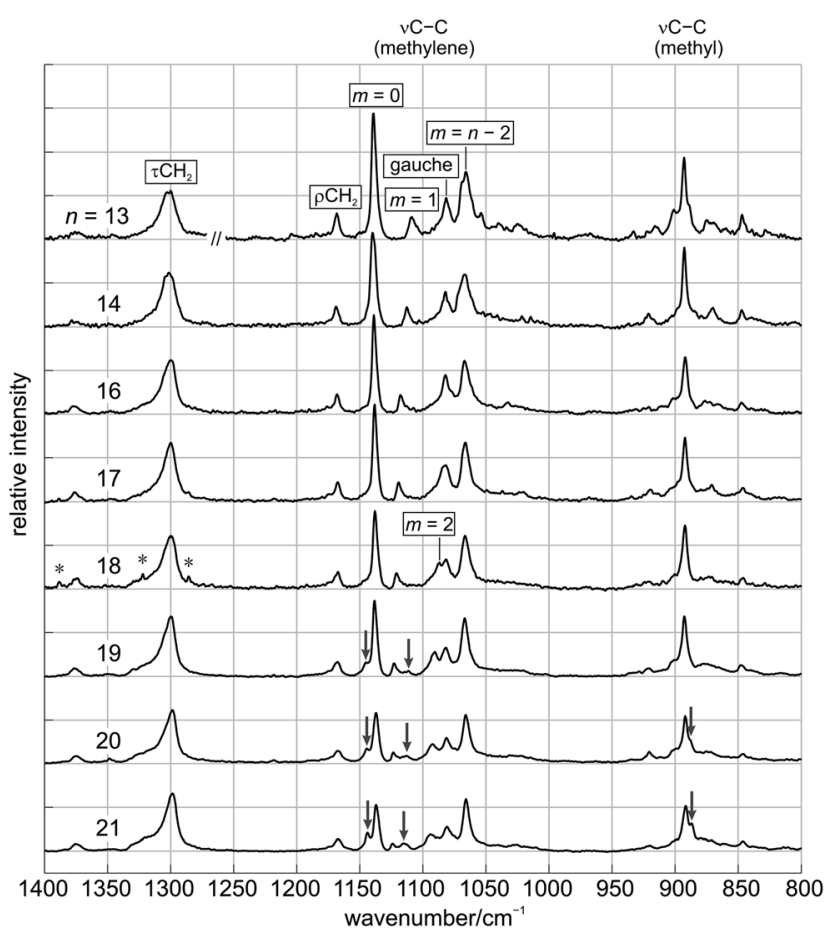

Fig. 9 Jet-cooled Raman spectra of the CC stretching region of alkanes with chain length $n=13,14$, and 16-21 in He expansions, scaled to the integral of the $\mathrm{CH}_{2}$ twisting vibration ( $\rho=$ rocking, $\nu=$ stretching, $\tau=$ twisting). Bands assigned to hairpin conformers are marked with arrows, impurities with *. (nodeless) and out-of-phase CC stretching vibrations ( $m=n-$ 2 ), whereas the band marked $m=1$ includes one node and moves as a function of chain length, ${ }^{64}$ in analogy to the LAM modes. Its Raman intensity is enhanced when a single gauche angle comes close to the central node and it can therefore serve as a marker for the decaying single-gauche fraction as a function of chain length, confirming the LAM observation. The $m=2$ mode shows a similar size dependence, although somewhat hidden in the stronger transitions. The slightly blue-shifting band series at $1111-1115 \mathrm{~cm}^{-1}$ and the stationary series at 1144 $\mathrm{cm}^{-1}$ are systematically shifted hairpin counterparts to the $m=2$ and $m=0$ series, which have in phase CC stretching character in both arms of the hairpin and phase switches pinned to the ggtgg kink. Contributions from disordered multiple gauche conformations are not expected to show such a clean spectral evolution.

The bands located near $900 \mathrm{~cm}^{-1}$ are associated with vibrations localized near the $-\mathrm{CH}_{3}$ end groups and sensitive to their conformation. ${ }^{\mathbf{8 4}}$ The concentration of intensity around 892 $\mathrm{cm}^{-1}$ (characteristic for $-\mathrm{tt}$ ends) in the jet spectra does not match the liquid phase situation ${ }^{62,86}$ and indicates low conformational disorder close to chain ends. The shoulder which this band develops in the jet spectra for $n=20,21$ is due to partially kink-localized vibrations in hairpin conformations. ${ }^{63}$ It coincidentally eclipses with the main - tt peak for $n=19$, as shown by simulations. There is thus threefold experimental evidence from the CC stretching spectra that hairpin formation is detectable at $n>18$, fully in line with the LAM spectral interpretation.

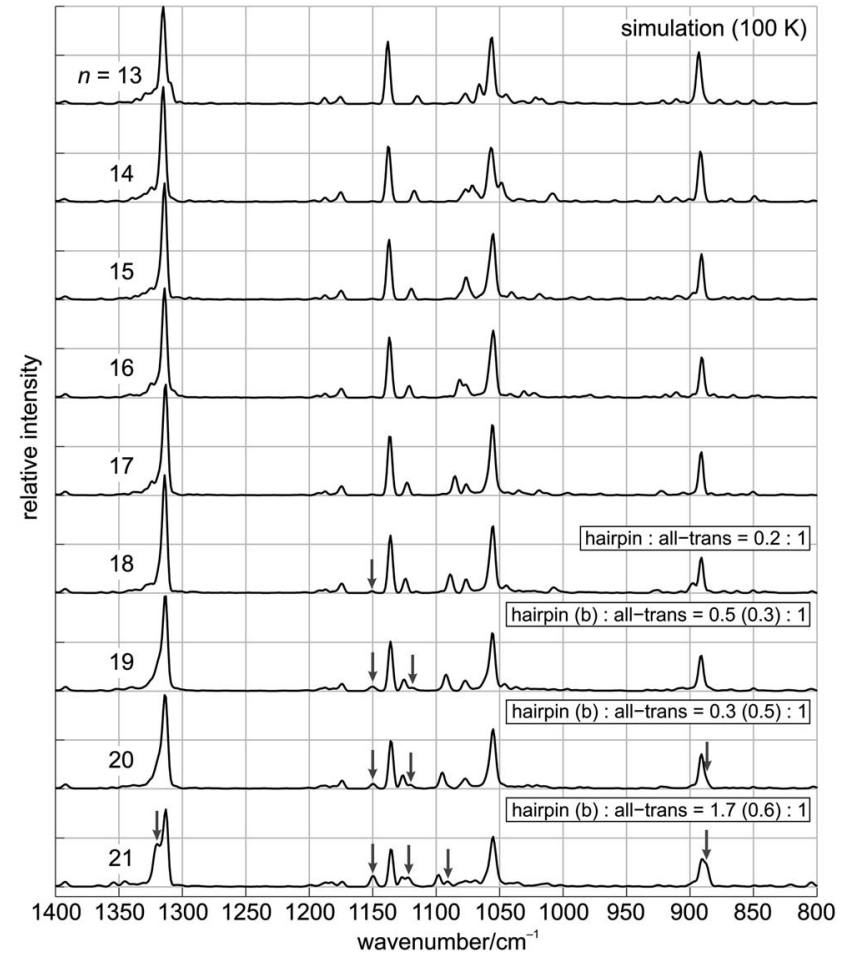

Fig. 10 Simulated Raman spectra of the $C C$ stretching region from harmonic B3LYP-D3/6-311++G** calculations (wavenumbers scaled by 0.99 ), see low-frequency spectra and simulations. Intensity scaled in analogy to the experimental spectra. 


\section{$8 \mathrm{CH}$ stretching manifold}

The $\mathrm{CH}$ stretching modes show a strong Raman visibility (Fig. 11), but due to their high energy, they also exhibit anharmonic complexity. ${ }^{3 \mathbf{3 1 5 2 , 8 7}}$ Nevertheless, conformational order is encoded. ${ }^{28,85,88}$ The overall band shape and four sharp peaks at $\approx 2860$ (symmetric methylene stretch), 2870 (symmetric methyl stretch), 2890 (asymmetric methylene stretch), and $2975 \mathrm{~cm}^{-1}$ (asymmetric methyl stretch) are common to all chain lengths, whereas beyond $n=17$, peak widths increase, two broad bands grow at $\approx 2908$ and $\approx 2925 \mathrm{~cm}^{-1}$, and the peak at $2860 \mathrm{~cm}^{-1}$ develops a low-frequency shoulder. The latter two spectral changes occur in a region where Fermi resonance between the symmetric methylene stretching fundamental and overtones of methylene bending modes is active, pushing the former band down and the latter up in wavenumber. ${ }^{87,88}$ In both cases, a detailed intensity analysis shows an accelerated build-up around $n=18-19$, relative to the conformationally insensitive asymmetric methyl stretching band. Harmonic analysis is impeded by the strong Fermi resonance, but it should be noted that methylene modes localized on the ggtgg kink are predicted in the center of the $\mathrm{CH}$ stretching manifold and condensed phase features are systematically $10-15 \mathrm{~cm}^{-1}$ lower in wavenumber than the jet spectra counterparts. The latter could explain the low frequency shoulder of the symmetric stretch, because the hairpin represents a first step into the condensed phase interactions. However, it would be difficult to separate this from a small extent of cluster formation.

Continuing on the phenomenological analysis, two spectral aspects show a clear switch around $n=17-18$. One is the width of the symmetric methyl stretching band at $2870 \mathrm{~cm}^{-1}$, which is constant up to $n=16$ and then starts to nearly double. For such a localized vibration, a close contact of the two end groups and thus hairpin formation is a suggestive explanation. The other is the peak intensity of the symmetric methylene stretch relative to the asymmetric methyl stretch. It steadily increases with $n$ as it should for more and more methylene groups in between the two methyl groups. This increase comes to a sudden halt at $n=18$, which is consistent with the emergence of a kink in the middle of the chain.

Thus, there is at least fourfold evidence for the onset of hairpin formation at or beyond $n=18$ in the $\mathrm{CH}$ stretching region, although the complexity of the vibrational dynamics ${ }^{52}$ enforces a more phenomenological analysis. This adds to the three-fold evidence in the CC stretching region and to the most direct evidence for hairpin structures in the longitudinal acoustic branch.

\section{Alkane folding}

The next step is to analyze the energy balance between the two competitors for the global minimum structure of alkanes - the all-trans and the hairpin structure.

Experimentally, it makes sense to estimate the all-trans fraction among all conformations as a function of chain length. This was done by monitoring the intensity ratio of the LAM-1 band to the $\mathrm{CH}$ stretching manifold, assuming that the $\mathrm{CH}$ scattering strength is relatively insensitive to conformation. Indeed, within the reproducibility of switching between the LAM-1 and $\mathrm{CH}$ stretching measurements, this is the case. Despite multiple sequential measurements to compensate for long term concentration drifts and careful realignment, there is an experimental scatter of up to $\pm 30 \%$ in the respective ratios. Therefore, it is not critical to use computed harmonic scattering strength ratios (about 0.1 between LAM-1 and the full $\mathrm{CH}$ stretching range) to convert the intensity ratio into an all-trans fraction in the expansion. Despite the $\pm 30 \%$ error bar, the results confirm that the all-trans fraction drops from $0.2-0.3$ for $n=13$ to $0.05-0.2$ for $n>18$, qualitatively in line with expectations (Fig. 3). The results also confirm that $\mathrm{CF}_{4}$ addition to the expansion lowers the conformational temperature from $\approx 120 \mathrm{~K}$ to $\approx 110 \mathrm{~K}$, when assuming an average gauche penalty of

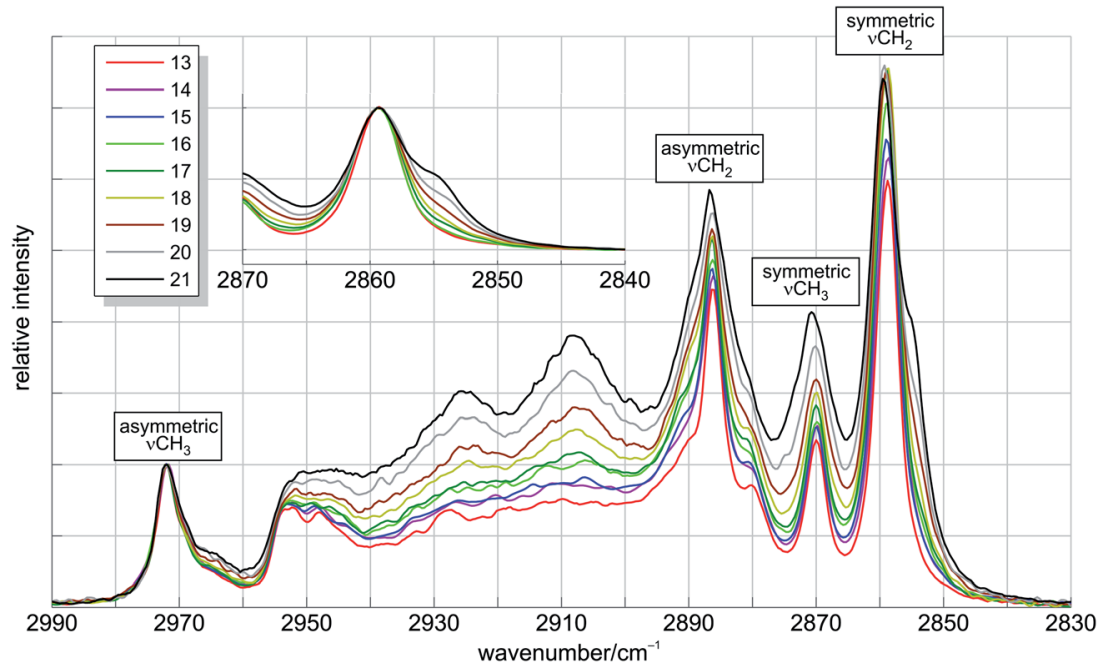

Fig. 11 Averaged Raman jet spectra of alkanes in He expansions in the $\mathrm{CH}$ stretching region, scaled to the asymmetric methyl stretching band at $\approx 2970 \mathrm{~cm}^{-1}$. The inlay illustrates the evolution of the symmetric methylene $\mathrm{CH}$ stretching band. 
$2.5 \mathrm{~kJ} \mathrm{~mol}^{-1}$, which by itself has an uncertainty and model error of $0.5 \mathrm{~kJ} \mathrm{~mol}^{-1}$. Consistent with this, the gain in all-trans signal relative to its spectral neighborhood upon addition of $\mathrm{CF}_{4}$ is (30 $\pm 10) \%$, without systematic chain length dependence. These findings clearly show that hairpin structures - undoubtedly present for $n>18$ - do not compete with all-trans structures in the supersonic jet expansion in a substantial way. This is actually quite helpful for the determination of the elastic modulus of an infinite polyethylene chain. Despite the possibility to extract Boltzmann-type temperatures between 80 and $110 \mathrm{~K}$ for single-gauche isomerization and $110 \pm 30 \mathrm{~K}$ for the alltrans fraction, this is not the case for quadruple gauche conversion to ggtgg in the supersonic jet expansion.

In those cases $(n=19-21)$ where two hairpin structures could be approximately quantified from the LAM spectra (with uncertainty similar to that of the absolute all-trans fraction), effective two-level temperatures can be estimated from the computed isomerization energies and the measured abundance. These are invariably close to or higher than $200 \mathrm{~K}$, even allowing for $30 \%$ error in their measured abundance. This shows that hairpin isomerization freezes at much higher temperature, which is reasonable, because the involved barrier for the required reptation along the contact line of the two arms is probably high. It also implies that hairpins are not in equilibrium with single-gauche conformations in the cold regions of the expansion. Conformations which have the potential to be relaxed to hairpin structures are quickly depleted and only single gauche-trans conversions continue further down the expansion. This translates into a kinetic delay of hairpin formation. They will only be observed in the expansion if they are already energetically competitive at $200 \mathrm{~K}$. Therefore, the observation threshold of $n=19$ (with abundance of $0.5+0.3$ relative to all-trans) is a rather strict upper bound for their energetical competitiveness at low temperature. This, and the optimum alignment of the two arms in the even-membered $n=$ 18 alkane, make it quite likely that the first alkane which has a hairpin global minimum is $n_{\mathrm{h}}=18$. Strictly speaking, experiment cannot rule out $n_{\mathrm{h}}=19$ and in terms of the $200 \mathrm{~K}$ conformational freezing limit, $n_{\mathrm{h}}=17$ would also be conceivable at least in principle. However, the steepness of the hairpin emergence points at $n_{\mathrm{h}}=18$ with an energy uncertainty of less than $R T \approx 2 \mathrm{~kJ} \mathrm{~mol}^{-1}$. Nevertheless, the indirect evidence for folded octadecane in the $\mathrm{CH}$ stretching region and the indications for early freezing of the folded conformations suggests $n_{\mathrm{h}}=18 \pm 1$ as a conservative experimental estimate from the present work.

At this point, a brief comparison to empirical force field and quantum mechanics predictions for the switching length $n_{\mathrm{h}}$ to hairpin structures is indicated., 2 ,23,25,89 The $n_{\mathrm{h}}$ values are summarized in Table 2, together with dispersion corrected DFT results from this work and the experimental result. In some theoretical work, ${ }^{23,89}$ a $x^{ \pm} g^{\mp} x^{ \pm}$folding sequence $(x g x$ in the following, $x: \tau \approx 95^{\circ}$ ) was used, which is significantly less stable (by $13 \mathrm{~kJ} \mathrm{~mol}^{-1}$ for $n=20$ on the B3LYP-D3/def2-TZVP level and by $12 \mathrm{~kJ} \mathrm{~mol}^{-1}$ for smaller alkanes at coupled cluster level ${ }^{8}$ ) but would be difficult to rule out from spectroscopic evidence alone. One should emphasize that the goal of that work was not to pinpoint the exact switching point, but to emphasize its existence and the importance of dispersion corrections to locate it.

Among the force fields, the performance of MM2 (ref. 90) and of the $n$-pentane-scaled OPLS-AA result ${ }^{7}$ (in parentheses) is remarkable. As all these force fields overestimate the ggtgg folding energy, the unscaled success of MM2 relies on an overestimate of the van der Waals attractions, which was indeed corrected in the later version MM3. ${ }^{4}$ We suggest to use the "hairpin benchmark" along with ab initio ggtgg folding energies in the parametrization process of future force field releases to yield more reliable conformer energies from such predictions.

The first-principles approach abbreviated MP2 + CC in the table was described in detail before. ${ }^{25}$ Structurally, it is very close to the MP2 basis set limit and energetically, it includes important higher order electron correlation contributions. It is one of the two highest level calculations available for these chain lengths and its only significant limitations may be the missing structural relaxation with higher order electron correlation and anharmonic contributions to the zero point energy. They give rise to the uncertainty included in $n_{\mathrm{h}}=18 \pm 1$. The resulting hairpin energies from this approach are given in Fig. 12. Very recently, another high level $a b$ initio study was published ${ }^{24}$ which confirms our experimental and the MP2 + CC calculations at canonical $\operatorname{CCSD}(\mathrm{T})$ level.

The B3LYP-D3 calculations from this work were intended to aid the spectral assignment, but their energetical predictions are also included in Fig. 12. The smaller Pople basis set predicts $n_{\mathrm{h}}=16$, the more complete def2-TZVP basis set $n_{\mathrm{h}}=17$, both in satisfactory agreement with experiment and high level theory. The good performance for shorter alkane conformational energies suggests that the agreement rests much less on error compensation than in the MM2 case. All accurate methods predict that the switch from an even chain length to the next shorter odd chain length raises the energy penalty of the hairpin by more than $2 \mathrm{~kJ} \mathrm{~mol}^{-1}$. Therefore, $n_{\mathrm{h}}=17$ rather than 18 (or 19) is a less likely interpretation of the experimental spectra despite their kinetically controlled nature. If heptadecane were

Table 2 Chain length prediction $n_{\mathrm{h}}$ for the first globally stable hairpin according to different computational methods and Raman jet spectra

\begin{tabular}{|c|c|c|}
\hline Method & Reference & Critical chain length \\
\hline MNDO & 22 & $\infty$ \\
\hline PM3 & 22 & 12 \\
\hline AM1 & 22 & 60 \\
\hline MM2 & 22 & 18 \\
\hline MM3 & 22 & 25 \\
\hline AMBER & 22 & 26 \\
\hline OPLS-AA & 7 & $22(16-18)^{a}$ \\
\hline $\mathrm{B} 97-\mathrm{D}^{b}$ & This work & 13 \\
\hline B3LYP-D3 $^{c}$ & This work & 16 \\
\hline B3LYP-D3 ${ }^{d}$ & This work & 17 \\
\hline CCSD FNO(T) & 24 & $\geq 16$ \\
\hline $\mathrm{MP} 2+\mathrm{CC}$ & 25 & $18 \pm 1$ \\
\hline Raman jet & This work & $18 \pm 1$ \\
\hline
\end{tabular}

${ }^{a}$ Estimated accounting for known deficiencies of OPLS-AA. ${ }^{7}{ }^{b} 6^{-}$ $311++\mathrm{G}(\mathrm{d}, \mathrm{p})$ basis set, Gaussian $09 .{ }^{45}{ }^{c} 6-311++\mathrm{G}^{* *}$ basis set, Turbomole v6.4. ${ }^{46} d$ def2-TZVP basis set, Turbomole v6.4. ${ }^{46}$ 


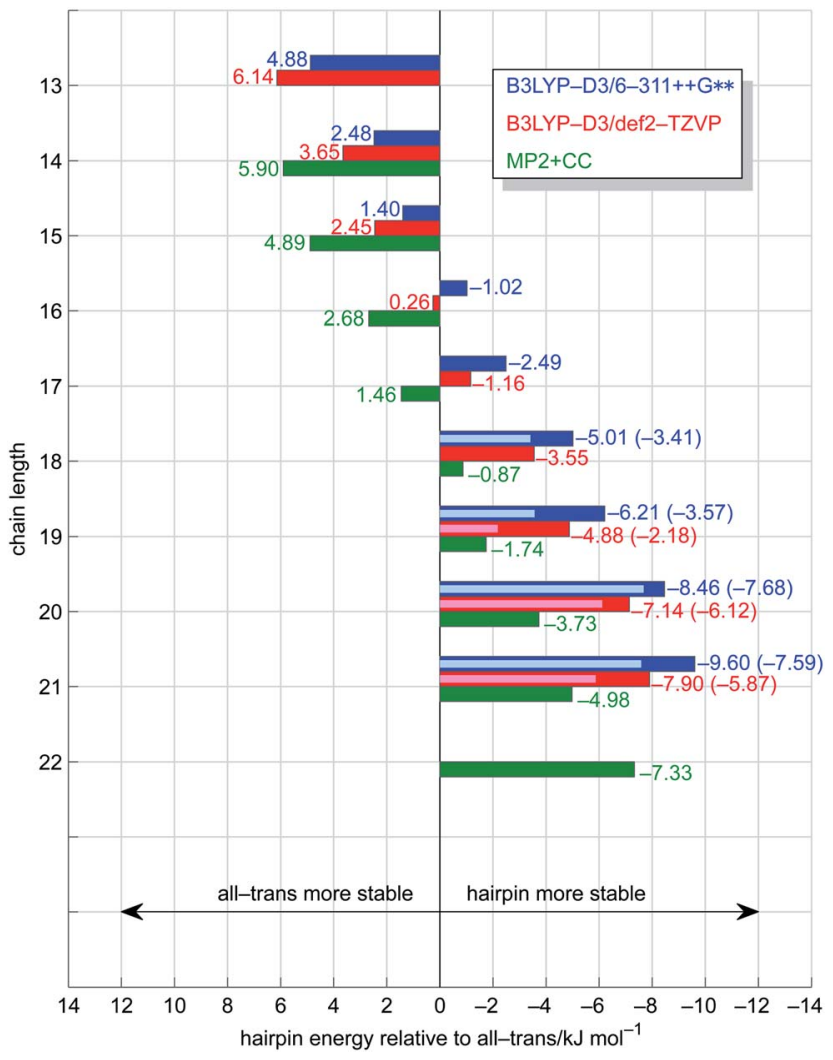

Fig. 12 Energy of the hairpin conformer relative to the all-trans conformer. Blue, red and green bars comprise electronic and zeropoint vibrational energy from B3LYP-D3/6-311++G**, B3LYP-D3/ def2-TZVP, and a composite MP2/coupled cluster calculation (MP2 + CC). ${ }^{25}$ Energies from hairpin $b$ conformers are drawn with light colors and are given in parentheses.

already more stable in the hairpin form, octadecane hairpins should survive at $200-300 \mathrm{~K}$ and give rise to a more substantial spectral signature, whereas the intensity gain towards nonadecane should be more moderate than observed. The reason is a moderate $\approx 1 \mathrm{~kJ} \mathrm{~mol}^{-1}$ relative energy gain predicted unanimously for this step (see Fig. 12). However, if future experiments manage to produce large populations of octadecane or even heptadecane hairpins, this statement would have to be challenged. More thermodynamically equilibrated experiments ${ }^{91}$ could bring more certainty. ${ }^{25}$

An interesting question is the solvent dependence of the hairpin folding threshold. Compared to vacuum, any polarizable environment is expected to compete with the self-solvation tendency and to shift the transition in first order to higher $n_{\mathrm{h}}$. The effect of water is discussed controversially due to its high cohesion..$^{22,92,93}$ Of course, the usually moderate temperature in solvents means that entropy aspects complicate the issue and a statistical analysis of various folded structures is necessary ${ }^{94}$ There is some indirect experimental NMR evidence in deuterated non-protic solvents ${ }^{95}$ that $n=21,22$ may show a different conformational distribution than shorter chains, but $n=17$ also exhibits some anomaly and its origin remains unclear. Calculations predict the folding in such solvents to be significantly more delayed..$^{22}$ This is in agreement with the solid state behaviour, which shows folding beyond about $n=150$. Even this may be kinetically controlled, as a majority of all-trans conformations can still be realized for $n=390$ if the crystallization is carried out very slowly ${ }^{12}$ or the crystals are annealed..$^{96}$ On the other hand, there is apparently only little solvent influence on alkane conformational distributions before the onset of folding. ${ }^{7}$

We close this section with a few curiosities. The hairpin is certainly not the final folding motive. It will be superseded by others like the paper-clip ${ }^{7,22}$ for longer chain lengths. Even knots appear to become feasible beyond $n=21,{ }^{97}$ but it will require much longer chains before such knots can possibly start to contribute to the global minimum structure. Experimentally, alkanes with $n>15$ are found to freeze at the surface before the bulk freezing point is reached. ${ }^{98}$ It is unlikely that this phenomenon is related to hairpin formation and indeed a similar phenomenon is observed for even shorter alkanes, when they are supercooled. ${ }^{99}$ The chain length of biological lipids is coincidentally close to the folding limit but this has little relevance for membranes, as membrane embedding shifts the folding point in a similar way as crystal formation. Finally, the commercial use of alkanes with $n=16,18$ as heat storage materials in buildings (Micronal®) is related to their melting point ${ }^{13}$ and not to explicit hairpin folding.

\section{Conclusions}

At room temperature and above, fully hydrogenated singlestranded carbon rods ( $n$-alkanes) in the nanometer length domain have several more or less randomly distributed (gauche) kinks in the otherwise energetically favored stretched (all-trans or zig-zag) conformation. Neighboring kinks with the same handedness are slightly favored due to London dispersion interactions between the two strands. By reducing the temperature, the stretched-out state starts to dominate the distribution up to a chain length of $(2.2 \pm 0.2) \mathrm{nm}(n=17 \pm 1)$. Beyond this length, starting at $(2.4 \pm 0.2) \mathrm{nm}(n=18 \pm 1)$, the globally stable conformation is a folded hairpin state with two pairs of neighboring kinks, all of the same handedness, separated by a single stretched segment. The fully stretched conformation survives in a metastable state, if the cooling is rapid, as it has to be to avoid aggregation. This enables the experimental determination of the elastic modulus for an infinite stretched hydrogenated carbon rod of $(305 \pm 5) \mathrm{GPa}$.

If a new hydrocarbon force field with gas phase applicability is proposed, one can now ask the fundamental and simple to answer question: what is its folding threshold $n_{\mathrm{h}}$ for $n$-alkanes? Error compensation between poor cohesive energy terms and poor kink energy penalties can give the correct answer for the wrong reason. Therefore, conformational energies for short alkanes must also be checked. Similarly, if the longitudinal acoustic mode frequency as a function of chain length and thus the elastic modulus $E$ is modelled correctly, this could still be due to error compensation between intramolecular stretching and bending force constants. More likely, a good performance in these two benchmark quantities, namely $n_{\mathrm{h}}$ close to 18 and $E$ 
close to $305 \mathrm{GPa}$, can be taken as evidence for the overall quality of the force field.

These experimental reference values have been extracted from more than half a dozen independent spectroscopic pieces of evidence using spontaneous Raman scattering in supersonic jet expansions and from a straightforward extrapolation of a linear plot of the longitudinally acoustic mode wavenumber $v s$. reciprocal chain length to the origin. They may be considered as robust benchmarks for any theoretical description of isolated polyethylene strands and their interaction.

Further insights could be gained by selective and full deuteration of the hydrocarbons and by imaging of the jet expansion close to the nozzle. ${ }^{35}$ The technique can now be extended to other synthetic polymer models such as perfluorinated and methyl-substituted alkanes. While it is a long way from the synthetic polymer folding addressed in this work to protein folding in biophysics, ${ }^{\mathbf{1 0 0}}$ the challenges are similar: how to overcome the associated barriers in finding the global minimum, how to take into account or actually remove the molecular environment for benchmark purposes, and how to detect folding motifs spectroscopically. The subtle alkane folding issue is more challenging for quantum theory and spectroscopic detection, whereas peptide folding in the gas phase may be more difficult to drive close to the thermodynamic limit.

\section{Acknowledgements}

The authors thank the mechanics and electronics workshops for their expertise in the construction of the Raman jet setup and the German Research Foundation (DFG Su 121/2) for valuable financial support. R. Mata has provided important quantum chemical benchmark calculations and P. Zielke and T. $\mathrm{N}$. Wassermann have been essential in the early experimental phase of this project.

\section{References}

1 M. Matsuo and C. Sawatari, Elastic Modulus of Polyethylene in the Crystal Chain Direction as Measured by X-ray Diffraction, Macromolecules, 1986, 19, 2036-2040.

2 J. B. Klauda, B. R. Brooks, A. D. MacKerell, R. M. Venable and R. W. Pastor, An $A b$ Initio Study on the Torsional Surface of Alkanes and Its Effect on Molecular Simulations of Alkanes and a DPPC Bilayer, J. Phys. Chem. $B, 2005,109,5300-5311$.

3 A. Stone, The Theory of Intermolecular Forces, Oxford University Press, Oxford, 2nd edn, 2013.

$4 \mathrm{~J}$. H. Lii and N. L. Allinger, Molecular mechanics. The MM3 force field for hydrocarbons. 3. The van der Waals' potentials and crystal data for aliphatic and aromatic hydrocarbons, J. Am. Chem. Soc., 1989, 111, 8576-8582.

5 Y. Mo, A Critical Analysis on the Rotation Barriers in Butane, J. Org. Chem., 2010, 75, 2733-2736.

$6 \mathrm{~W}$. L. Jorgensen, D. S. Maxwell and J. Tirado-Rives, Development and Testing of the OPLS All-Atom Force
Field on Conformational Energetics and Properties of Organic Liquids, J. Am. Chem. Soc., 1996, 118, 11225-11236.

7 L. L. Thomas, T. J. Christakis and W. L. Jorgensen, Conformation of Alkanes in the Gas Phase and Pure Liquides, J. Phys. Chem. B, 2006, 110, 21198-21204.

8 D. Gruzman, A. Karton and J. M. L. Martin, Performance of $A b$ Initio and Density Functional Methods for Conformational Equilibria of $\mathrm{C}_{n} \mathrm{H}_{2 n+2}$ Alkane Isomers $(n=$ 4-8), J. Phys. Chem. A, 2009, 113, 11974-11983.

9 W. A. Herrebout, B. J. van der Veken, A. Wang and J. R. Durig, Enthalpy Difference between Conformers of $n$-Butane and the Potential Function Governing Conformational Interchange, J. Phys. Chem., 1995, 99, 578-585.

10 R. M. Balabin, Enthalpy Difference between Conformations of Normal Alkanes: Raman Spectroscopy Study of $n$-Pentane and $n$-Butane, J. Phys. Chem. A, 2009, 113, 1012-1019.

11 J. M. L. Martin, What Can We Learn about Dispersion from the Conformer Surface of $n$-Pentane, J. Phys. Chem. A, 2013, 117, 3118-3132.

12 G. Ungar, J. Stejny, A. Keller, I. Bidd and M. C. Whiting, The Crystallization of Ultralong Normal Paraffins: The Onset of Chain Folding, Science, 1985, 229, 386-389.

13 N. Sarier and E. Onder, Organic phase change materials and their textile applications: An overview, Thermochim. Acta, 2012, 540, 7-60.

14 L. L. Böhm, The Ethylene Polymerization with Ziegler Catalysts: Fifty Years after the Discovery, Angew. Chem., Int. Ed., 2003, 42, 5010-5030.

15 A. J. Peacock and L. Mandelkern, The Mechanical Properties of Random Copolymers of Ethylene: ForceElongation Relations, J. Polym. Sci., Part B: Polym. Phys., 1990, 28, 1917-1941.

16 G. R. Strobl and R. Eckel, A Raman spectroscopic determination of the interlamellar forces in crystalline $n$ alkanes and of the limiting elastic modulus $E_{\mathrm{c}}$ of polyethylene, J. Polym. Sci., Polym. Phys. Ed., 1976, 14, 913-920.

17 P. J. Barham and A. Keller, The achievement of highmodulus polyethylene fibers and the modulus of polyethylene crystals, J. Polym. Sci., Polym. Lett. Ed., 1979, 17, 591-593.

18 B. Fanconi and J. F. Rabolt, The determination of longitudinal crystal moduli in polymers by spectroscopic methods, J. Polym. Sci., Polym. Phys. Ed., 1985, 23, 12011215.

19 R. G. Snyder, H. L. Strauss, R. Alamo and L. Mandelkern, Chain-length dependence of interlayer interaction in crystalline $n$-alkanes from Raman longitudinal acoustic mode measurements, J. Chem. Phys., 1994, 100, 5422-5431.

20 G. D. Barrera, S. F. Parker, A. J. Ramirez-Cuesta and P. C. H. Mitchell, The Vibrational Spectrum and Ultimate Modulus of Polyethylene, Macromolecules, 2006, 39, 26832690.

21 T. N. Wassermann, J. Thelemann, P. Zielke and M. A. Suhm, The stiffness of a fully stretched polyethylene chain: A Raman jet spectroscopy extrapolation, J. Chem. Phys., 2009, 131, 161108. 
$22 \mathrm{~J}$. M. Goodman, What Is the Longest Unbranched Alkanewith a Linear Global Minimum Conformation?, J. Chem. Inf. Comput. Sci., 1997, 37, 876-878.

23 S. Grimme, J. Antony, T. Schwabe and C. Mück-Lichtenfeld, Density functional theory with dispersion corrections for supramolecular structures, aggregates, and complexes of (bio)organic molecules, Org. Biomol. Chem., 2007, 5, 741-758.

24 J. N. Byrd, R. J. Bartlett and J. A. Montgomery, At What Chain Length Do Unbranched Alkanes Prefer Folded Conformations?, J. Phys. Chem. A, 2014, 118, 1706-1712.

25 N. O. B. Lüttschwager, T. N. Wassermann, R. A. Mata and M. A. Suhm, The Last Globally Stable Extended Alkane, Angew. Chem., Int. Ed., 2013, 52, 463-466.

26 C. D. Sherrill, T. Takatani and E. G. Hohenstein, An Assessment of Theoretical Methods for Nonbonded Interactions: Comparison to Complete Basis Set Limit Coupled-Cluster Potential Energy Curves for the Benzene Dimer, the Methane Dimer, Benzene-Methane, and Benzene- $\mathrm{H}_{2} \mathrm{~S}$, J. Phys. Chem. A, 2009, 113, 10146-10159.

27 R. Eckel, M. Buback and G. Strobl, Untersuchung der druckinduzierten Kristallisation von Polyäthylen mit Hilfe einer neuen Raman-Hochdruckzelle, Colloid Polym. Sci., 1981, 259, 326-334.

28 R. G. Snyder, On Raman evidence for conformational order in liquid $n$-alkanes, J. Chem. Phys., 1982, 76, 3342-3343.

29 L. Brambilla and G. Zerbi, Local Order in Liquid n-Alkanes: Evidence from Raman Spectroscopic Study, Macromolecules, 2005, 38, 3327-3333.

$30 \mathrm{H}$. W. Schrötter, Update of reviews on Raman spectra of gases. I. Linear Raman spectroscopy, J. Mol. Struct., 2003, 661-662, 465-468.

31 N. A. Atamas, A. M. Yaremko, T. Seeger, A. Leipertz, A. Bienko, Z. Latajka, H. Ratajczak and A. J. Barnes, A study of the Raman spectra of alkanes in the Fermiresonance region, J. Mol. Struct., 2004, 708, 189-195.

32 I. F. Silvera and F. Tommasini, Intracavity Raman Scattering from Molecular Beams: Direct Determination of Local Properties in an Expanding Jet Beam, Phys. Rev. Lett., 1976, 37, 136-140.

33 D. H. Levy, Laser Spectroscopy of Cold Gas-Phase Molecules, Annu. Rev. Phys. Chem., 1980, 31, 197-225.

34 C. M. Lovejoy and D. J. Nesbitt, Slit pulsed valve for generation of long-path-length supersonic expansions, Rev. Sci. Instrum., 1987, 58, 807-811.

35 J. Zischang and M. A. Suhm, Infrared absorption imaging of 2D supersonic jet expansions: Free expansion, cluster formation, and shock wave patterns, J. Chem. Phys., 2013, 139, 024201.

36 M. Albrecht, C. A. Rice and M. A. Suhm, Elementary Peptide Motifs in the Gas Phase: FTIR Aggregation Study of Formamide, Acetamide, $\mathrm{N}$-Methylformamide, and $\mathrm{N}$ Methylacetamide, J. Phys. Chem. A, 2008, 112, 7530-7542.

37 R. M. Balabin, Conformational Equilibrium in Glycine: Experimental Jet-Cooled Raman Spectrum, J. Phys. Chem. Lett., 2010, 1, 20-23.

38 R. S. Ruoff, T. D. Klots, T. Emilsson and H. S. Gutowsky, Relaxation of conformers and isomers in seeded supersonic jets of inert gases, J. Chem. Phys., 1990, 93, 3142-3150.

39 U. Erlekam, M. Frankowski, G. von Helden and G. Meijer, Cold collisions catalyse conformational conversion, Phys. Chem. Chem. Phys., 2007, 9, 3786-3789.

40 W. Y. Sohn, J. S. Kang, S. Y. Lee and H. Kang, Fluorescence excitation spectrum and solvent-assisted conformational isomerization (SACI) of jet-cooled acetaminophen, Chem. Phys. Lett., 2013, 581, 36-41.

41 P. Zielke and M. A. Suhm, Concerted proton motion in hydrogen-bonded trimers: A spontaneous Raman scattering perspective, Phys. Chem. Chem. Phys., 2006, 8, 2826-2830.

42 N. O. B. Lüttschwager, T. N. Wassermann, S. Coussan and M. A. Suhm, Periodic bond breaking and making in the electronic ground state on a sub-picosecond timescale: $\mathrm{OH}$ bending spectroscopy of malonaldehyde in the frequency domain at low temperature, Phys. Chem. Chem. Phys., 2010, 12, 8201-8207.

43 R. G. Snyder, The structure of chain molecules in the liquid state: Low-frequency Raman spectra of $n$-alkanes and perfluoro-n-alkanes, J. Chem. Phys., 1982, 76, 3921-3927.

44 R. F. Schaufele and T. Shimanouchi, Longitudinal Acoustical Vibrations of Finite Polymethylene Chains, J. Chem. Phys., 1967, 47, 3605-3610.

45 M. J. Frisch, G. W. Trucks, H. B. Schlegel, G. E. Scuseria, M. A. Robb, J. R. Cheeseman, G. Scalmani, V. Barone, B. Mennucci, G. A. Petersson, H. Nakatsuji, M. Caricato, X. Li, H. P. Hratchian, A. F. Izmaylov, J. Bloino, G. Zheng, J. L. Sonnenberg, M. Hada, M. Ehara, K. Toyota, R. Fukuda, J. Hasegawa, M. Ishida, T. Nakajima, Y. Honda, O. Kitao, H. Nakai, T. Vreven, J. A. Montgomery, Jr, J. E. Peralta, F. Ogliaro, M. Bearpark, J. J. Heyd, E. Brothers, K. N. Kudin, V. N. Staroverov, R. Kobayashi, J. Normand, K. Raghavachari, A. Rendell, J. C. Burant, S. S. Iyengar, J. Tomasi, M. Cossi, N. Rega, J. M. Millam, M. Klene, J. E. Knox, J. B. Cross, V. Bakken, C. Adamo, J. Jaramillo, R. Gomperts, R. E. Stratmann, O. Yazyev, A. J. Austin, R. Cammi, C. Pomelli, J. W. Ochterski, R. L. Martin, K. Morokuma, V. G. Zakrzewski, G. A. Voth, P. Salvador, J. J. Dannenberg, S. Dapprich, A. D. Daniels, O. Farkas, J. B. Foresman, J. V. Ortiz, J. Cioslowski and D. J. Fox, Gaussian 09, Revision A.02, Gaussian, Inc., Wallingford CT, 2009.

46 TURBOMOLE v6.4 2012, a development of University of Karlsruhe and Forschungszentrum Karlsruhe GmbH, 19892007, TURBOMOLE GmbH, since 2007; available from http://www.turbomole.com.

47 T. N. Wassermann and M. A. Suhm, Ethanol Monomers and Dimers Revisited: A Raman Study of Conformational Preferences and Argon Nanocoating Effects, J. Phys. Chem. $A, 2010,114,8223-8233$.

48 S. Fitzwater and L. S. Bartell, Vapor-Phase Structure and Conformation of a Long-Chain n-Alkane. An Electron Diffraction Study, J. Am. Chem. Soc., 1976, 98, 8338-8344.

49 E. Funck, Symmetrie und Anzahl der Rotationsisomere von unverzweigten Polymerketten, Z. Elektrochem., 1958, 62, 901-905. 
50 G. Tasi, F. Mizukami, J. Csontos, W. Győrffy and I. Pálinkó, Quantum algebraic-combinatoric study of the conformational properties of $n$-alkanes. II, J. Math. Chem., 2000, 27, 191-199.

51 S. M. Long, T. T. Tran, P. Adams, P. Darwen and M. L. Smythe, Conformational searching using a population-based incremental learning algorithm, $J$. Comput. Chem., 2011, 32, 1541-1549.

52 J. Šebek, L. Pele, E. O. Potma and R. Benny Gerber, Raman spectra of long chain hydrocarbons: anharmonic calculations, experiment and implications for imaging of biomembranes, Phys. Chem. Chem. Phys., 2011, 13, 1272412733.

53 P. J. Flory, Foundations of Rotational Isomeric State Theory and General Methods for Generating Configurational Averages, Macromolecules, 1974, 7, 381-392.

54 P. J. Flory and R. L. Jernigan, Second and Fourth Moments of Chain Molecules, J. Chem. Phys., 1965, 42, 3509-3519.

55 A. Abe, R. L. Jernigan and P. J. Flory, Conformational Energies of $n$-Alkanes and the Random Configuration of Higher Homologs Including Polymethylene, J. Am. Chem. Soc., 1966, 88, 631-639.

56 S. Tsuzuki, L. Schäfer, H. Gotō, E. D. Jemmis, H. Hosoya, K. Siam, K. Tanabe and E. Ōsawa, Investigation of Intramolecular Interactions in n-Alkanes. Cooperative Energy Increments Associated with GG and GTG' Sequences, J. Am. Chem. Soc., 1991, 113, 4665-4671.

57 J. Dale, Conformational Aspects of Many-Membered Rings, Angew. Chem., Int. Ed., 1966, 5, 1000-1021.

58 H.-P. Grossmann and H. Bölstler, Longitudinal acoustic modes in cycloalkanes, Polym. Bull., 1981, 5, 175-177.

59 A. Zehnacker and M. A. Suhm, Chirality Recognition between Neutral Molecules in the Gas Phase, Angew. Chem., Int. Ed., 2008, 47, 6970-6992.

60 R. Zbinden, Infrared spectroscopy of high polymers, Academic Press, New York, 1964.

61 Modern Polymer Spectroscopy, ed. G. Zerbi, Wiley-VCH, Weinheim, 1999.

62 D. A. Cates, H. L. Strauss and R. G. Snyder, Vibrational Modes of Liquid n-Alkanes: Simulated Isotropic Raman Spectra and Band Progressions for $\mathrm{C}_{5} \mathrm{H}_{12}-\mathrm{C}_{20} \mathrm{H}_{42}$ and $\mathrm{C}_{16} \mathrm{D}_{34}$, J. Phys. Chem., 1994, 98, 4482-4488.

63 G. Zerbi and M. Gussoni, Defect modes for (200), GGTGG, tight fold re-entry in polyethylene single crystals, Polymer, 1980, 21, 1129-1134.

64 R. G. Snyder and J. H. Schachtschneider, Vibrational analysis of the n-paraffins - I: Assignments of infrared bands in the spectra of $\mathrm{C}_{3} \mathrm{H}_{8}$ through $n-\mathrm{C}_{19} \mathrm{H}_{40}$, Spectrochim. Acta, 1963, 19, 85-116.

65 T. Shimanouchi, Local and overall vibrations of polymer chains, Pure Appl. Chem., 1973, 36, 93-108.

66 M. D. Wodrich, C. Corminboeuf and P. von Ragué Schleyer, Systematic Errors in Computed Alkane Energies Using B3LYP and Other Popular DFT Functionals, Organic Letters, 2006, 8, 3631-3634.

67 S. Grimme, J. Antony, S. Ehrlich and H. Krieg, A consistent and accurate $a b$ initio parametrization of density functional dispersion correction (DFT-D) for the 94 elements $\mathrm{H}-\mathrm{Pu}, J$. Chem. Phys., 2010, 132, 154104.

68 F. Weigend, M. Häser, H. Patzelt and R. Ahlrichs, RI-MP2: optimized auxiliary basis sets and demonstration of efficiency, Chem. Phys. Lett., 1998, 294, 143-152.

69 K. Eichkorn, F. Weigend, O. Treutler and R. Ahlrichs, Auxiliary basis sets for main row atoms and transition metals and their use to approximate Coulomb potentials, Theor. Chem. Acc., 1997, 97, 119-124.

70 E. E. Zvereva, A. R. Shagidullin and S. A. Katsyuba, Ab Initio and DFT Predictions of Infrared Intensities and Raman Activities, J. Phys. Chem. A, 2011, 115, 63-69.

71 J. Neugebauer, M. Reiher and B. A. Hess, Coupled-cluster Raman intensities: Assessment and comparison with multiconfiguration and density functional methods, $J$. Chem. Phys., 2002, 117, 8623-8633.

72 S.-I. Mizushima and T. Simanouti, Raman Frequencies of $n$ Paraffin Molecules, J. Am. Chem. Soc., 1949, 71, 1320-1324.

73 I. Sakurada, T. Ito and K. Nakamae, Elastic moduli of the crystal lattices of polymers, J. Polym. Sci., Part C: Polym. Symp., 1967, 15, 75-91.

74 J. F. Rabolt and B. Fanconi, Longitudinal acoustic modes of polytetrafluoroethylene copolymers and oligomers, Polymer, 1977, 18, 1258-1264.

75 J. F. Rabolt and B. Fanconi, Longitudinal acoustic mode in helical polymers: Poly(oxymethylene) and isotactic polypropylene, J. Polym. Sci., Polym. Lett. Ed., 1977, 15, 121-127.

76 A. Karpfen, $A b$ initio studies on polymers. V. All-transpolyethylene, J. Chem. Phys., 1981, 75, 238-245.

77 S. Suhai, $A b$ initio calculation of polyethylene deformation including electron correlation effects, J. Polym. Sci., Polym. Phys. Ed., 1983, 21, 1341-1346.

78 J. C. L. Hageman, R. J. Meier, M. Heinemann and R. A. de Groot, Young Modulus of Crystalline Polyethylene from Ab Initio Molecular Dynamics, Macromolecules, 1997, 30, 5953-5957.

79 C. W. Bunn, The crystal structure of long-chain normal paraffin hydrocarbons. The "shape" of the $>\mathrm{CH}_{2}$ group, Trans. Faraday Soc., 1939, 35, 482-491.

80 D. J. Lacks and G. C. Rutledge, Simulation of the temperature dependence of mechanical properties of polyethylene, J. Phys. Chem., 1994, 98, 1222-1231.

81 R. R. Laher and F. R. Gilmore, Improved Fits for the Vibrational and Rotational Constants of Many States of Nitrogen and Oxygen, Phys. Chem. Ref. Data, 1991, 20, 685-712.

82 V. Boudon, J. Mitchell, A. Domanskaya, C. Maul, R. Georges, A. Benidar and W. G. Harter, High-resolution spectroscopy and analysis of the $\nu_{3} 2 \nu_{4}$ dyad of $\mathrm{CF}_{4}$, Molecular Physics, 2011, 109, 2273-2290.

83 A. L. Brower, J. R. Sabin, B. Crist and M. A. Ratner, Ab initio molecular orbital studies of polyethylene deformation, Int. J. Quantum Chem., 1980, 18, 651-654.

84 M. Maroncelli, S. P. Qi, H. L. Strauss and R. G. Snyder, Nonplanar conformers and the phase behavior of solid $n$ alkanes, J. Am. Chem. Soc., 1982, 104, 6237-6247. 
85 C. J. Orendorff, M. W. Ducey, Jr and J. E. Pemberton, Quantitative Correlation of Raman Spectral Indicators in Determining Conformational Order in Alkyl Chains, $J$. Phys. Chem. A, 2002, 106, 6991-6998.

86 G. Zerbi, R. Magni, M. Gussoni, K. H. Moritz, A. Bigotto and S. Dirlikov, Molecular mechanics for phase transition and melting of $n$-alkanes: A spectroscopic study of molecular mobility of solid n-nonadecane, J. Chem. Phys., 1981, 75, 3175-3194.

87 R. G. Snyder and J. R. Scherer, Band structure in the C-H stretching region of the Raman spectrum of the extended polymethylene chain: Influence of Fermi resonance, $J$. Chem. Phys., 1979, 71, 3221-3228.

88 R. G. Snyder, H. L. Strauss and C. A. Elliger, Carbonhydrogen stretching modes and the structure of $n$-alkyl chains. 1. Long, disordered chains, J. Phys. Chem., 1982, 86, 5145-5150.

89 T. Schwabe and S. Grimme, Double-hybrid density functionals with long-range dispersion corrections: higher accuracy and extended applicability, Phys. Chem. Chem. Phys., 2007, 9, 3397-3406.

90 N. L. Allinger, Conformational analysis. 130. MM2. A hydrocarbon force field utilizing V1 and V2 torsional terms, J. Am. Chem. Soc., 1977, 99, 8127-8134.

91 N. R. Hutzler, H.-I. Lu and J. M. Doyle, The Buffer Gas Beam: An Intense, Cold, and Slow Source for Atoms and Molecules, Chem. Rev., 2012, 112, 48034827.

92 A. Wallqvist and D. G. Covell, Free-Energy Cost of Bending $n$-Dodecane in Aqueous Solution. Influence of the
Hydrophobic Effect and Solvent Exposed Area, J. Phys. Chem., 1995, 99, 13118-13125.

93 A. L. Ferguson, P. G. Debenedetti and A. Z. Panagiotopoulos, Solubility and Molecular Conformations of $n$-Alkane Chains in Water, J. Phys. Chem. B, 2009, 113, 6405-6414.

94 S. Chakrabarty and B. Bagchi, Self-Organization of n-Alkane Chains in Water: Length Dependent Crossover from Helix and Toroid to Molten Globule, J. Phys. Chem. B, 2009, 113, 8446-8448.

95 L. B. Alemany, Additional insights from very-highresolution ${ }^{13} \mathrm{C}$ NMR spectra of long-chain $n$-alkanes, Magn. Reson. Chem., 2013, 51, 605-613.

96 S. N. Magonov, N. A. Yerina, G. Ungar, D. H. Reneker and D. A. Ivanov, Chain Unfolding in Single Crystals of Ultralong Alkane $\mathrm{C}_{390} \mathrm{H}_{782}$ and Polyethylene: An Atomic Force Microscopy Study, Macromolecules, 2003, 36, 5637-5649.

97 C. S. Krinas and I. N. Demetropoulos, $\mathrm{C}_{22} \mathrm{H}_{46}$ : The smallest open 3 -knotted alkane by computer-aided design, J. Mol. Graphics Modell., 2008, 26, 1153-1159.

98 B. M. Ocko, X. Z. Wu, E. B. Sirota, S. K. Sinha, O. Gang and M. Deutsch, Surface freezing in chain molecules: Normal alkanes, Phys. Rev. E: Stat., Nonlinear, Soft Matter Phys., 1997, 55, 3164-3182.

99 V. P. Modak, H. Pathak, M. Thayer, S. J. Singer and B. E. Wyslouzil, Experimental evidence for surface freezing in supercooled n-alkane nanodroplets, Phys. Chem. Chem. Phys., 2013, 15, 6783-6795.

100 C. M. Dobson, Protein folding and misfolding, Nature, 2003, 426, 884-890. 